\title{
Nonlinear Controllers for a Light-Weighted All-Electric Vehicle Using Chebyshev Neural Network
}

\author{
Vikas Sharma and Shubhi Purwar \\ Department of Electrical Engineering, Motilal Nehru National Institute of Technology, Allahabad 211004, India \\ Correspondence should be addressed to Vikas Sharma; vikassharma.ecb@gmail.com
}

Received 24 October 2013; Revised 19 March 2014; Accepted 20 March 2014; Published 22 April 2014

Academic Editor: Tang-Hsien Chang

Copyright ( 2014 V. Sharma and S. Purwar. This is an open access article distributed under the Creative Commons Attribution License, which permits unrestricted use, distribution, and reproduction in any medium, provided the original work is properly cited.

Two nonlinear controllers are proposed for a light-weighted all-electric vehicle: Chebyshev neural network based backstepping controller and Chebyshev neural network based optimal adaptive controller. The electric vehicle (EV) is driven by DC motor. Both the controllers use Chebyshev neural network (CNN) to estimate the unknown nonlinearities. The unknown nonlinearities arise as it is not possible to precisely model the dynamics of an EV. Mass of passengers, resistance in the armature winding of the DC motor, aerodynamic drag coefficient and rolling resistance coefficient are assumed to be varying with time. The learning algorithms are derived from Lyapunov stability analysis, so that system-tracking stability and error convergence can be assured in the closed-loop system. The control algorithms for the EV system are developed and a driving cycle test is performed to test the control performance. The effectiveness of the proposed controllers is shown through simulation results.

\section{Introduction}

In recent years due to stricter emission standards and global fuel supply issues, researchers in the automobile industry are paying more attention to zero-polluting electric vehicles (EVs) for energy conservation and environmental protection. EV has emerged as a promising alternative to improve fuel economy while meeting the tightened emission standards [1]. $\mathrm{EV}$ is used in many applications, particularly for the purpose of patrolling and other short-range transportations. A lot of work has been reported in the literature for reducing the cost, extending the driving range, and sophisticated energy management strategies to improve the performance and usage of energy [2-6]. Direct current (DC) power is supplied from the battery, and therefore EVs driven by DC motor are a favorable selection. The control of DC motor is simple and it can provide comparatively larger startup torque. In addition to the primary function of propulsion, the DC motor can also be used effectively as the braking device because of its fast torque response characteristics and capability of regeneration $[7,8]$.

The dynamics of EV are inherently nonlinear and it is well known that nonlinear control techniques are superior to the conventional linear control methods $[9,10]$. The performance of nonlinear control techniques, specifically, the differentialgeometric approach, to the control of EV is presented in [11]. Due to the complex operating conditions of $\mathrm{EV}$, intelligent or fuzzy control is suggested in $[12,13]$. In [12], the fuzzy logic controller (FLC) is modeled to be capable of increasing the initial torque required for the electric vehicle drive with variable speed characteristics and high efficiency. In [13], fuzzy logic based controller to control the wheel slip for electric vehicle antilock braking systems (ABSs) is developed. A methodology to generate stochastic drive cycles for the design and control optimization of EVs is detailed in [14]. An algorithm for determining online energy based driving guidance for an EV using particle swarm optimization is developed in [15].

Neural networks have been used for approximation of nonlinear systems, for classification of signals and for associative memory. Chebyshev neural network (CNN) has been shown to be able to approximate any continuous functions over a compact set to arbitrary accuracy [16-18]. $\mathrm{CNN}$ is a functional link neural network (FLN) based on Chebyshev polynomials. The efficacy of CNN in the areas of online system identification $[19,20]$ and tracking controller 
for nonlinear systems has been established [21-23]. The proposed controller does not necessitate exact knowledge of the unknown nonlinearities. The CNN is used for estimating the unknown nonlinearities of the system. The adaptation laws for the $\mathrm{CNN}$ weights are such that they guarantee the stability of the system. The tracking error mainly depends on the CNN, feedback functions to be used for the weight adaptation law, and other design parameters.

It is not possible to precisely model the dynamics of an $\mathrm{EV}$ as some parameters may vary with time/conditions. For example, the resistance in the armature winding of the DC motor changes as the temperature varies and the aerodynamic drag coefficient $C_{d}$ and the rolling resistance coefficient $\mu_{r r}$ are varying because of wind and road conditions, respectively. In this paper, the resistance in the armature winding $\left(R_{a}\right)$ of the DC motor, the aerodynamic drag coefficient $C_{d}$, the rolling resistance coefficient $\mu_{r r}$, and the mass of the passengers $(\Delta M)$ are considered to be varying with time resulting in unknown nonlinearities. The aim of this paper is to design $\mathrm{CNN}$ based backstepping controller and $\mathrm{CNN}$ based optimal adaptive controller for EV in the presence of unknown nonlinearities and test the performance of the overall system on NEDC drive cycle test.

The paper is organized as follows. In Section 2, the description of the complete EV system and the structure of $\mathrm{CNN}$ will be presented. The problem statement is introduced in Section 3. The design of conventional backstepping controller and CNN based backstepping controller is described in Section 4. In Section 5, we give an optimal control design for EV systems using the H-J-B equation followed by a CNN based optimal adaptive controller. Section 6 validates the performance of the proposed controllers through simulations and drive cycle test followed by conclusion.

\section{EV System Description and CNN Structure}

An EV system dynamics mainly comprises two parts: the vehicle dynamics and dynamics of the motor system, as shown in Figure 1. Motor system is connected to EV system through transmission unit, which includes the gearing system. In the actual EVs, the driver provides the command signal through the accelerator/brake pedal in the form of acceleration/deceleration to the controller of the propulsion system. The DC motor is used in the proposed EV system for propulsion and DC motor system is connected to EV system through transmission unit, which includes the gearing system. Accordingly, the speed of DC motor is controlled so as to control the actual EV system.

2.1. Vehicle Dynamics. The major factors that affect the vehicle dynamics are road condition, aerodynamic drag, hill climbing, acceleration, and so forth. After these factors are taken into account, vehicle dynamics can be written as follows [1]:

$$
F=\underbrace{\mu_{r r} m g}_{1}+\underbrace{\frac{1}{2} \rho A C_{d} v^{2}}_{2}+\underbrace{m g \sin \phi}_{3}+\underbrace{m \frac{d v}{d t}}_{4},
$$

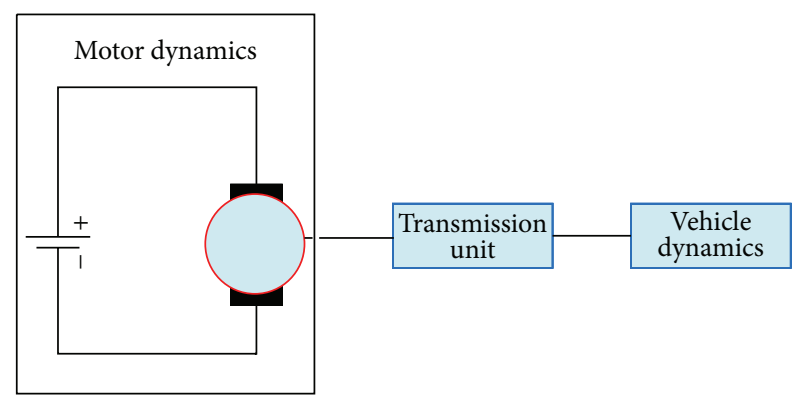

Figure 1: EV system.

where $\mu_{r r}$ is the rolling resistance coefficient, $m$ is the mass of the EV, $g$ is the gravity acceleration, $\rho$ is the air density, $A$ is the frontal area of the vehicle, $C_{d}$ is the drag coefficient, $v$ is the driving velocity of the vehicle, and $\phi$ is the hill climbing angle. In this paper, the aerodynamic $\operatorname{drag}$ coefficient $C_{d}$ and the rolling resistance coefficient $\mu_{r r}$ are assumed to be varying with time. In [1] $m$ is a constant which is a very stringent assumption. In the proposed work $m$ includes the mass of vehicle $M$ and the mass of passengers $\Delta M$; that is, $m=$ $M+\Delta M$. Thus $m$ is varying with time and not a constant.

In the vehicle dynamics (1), the first term assimilates to the rolling resistance force; the second term assimilates to the aerodynamic drag force; the third term assimilates to the hill climbing force; and the fourth term assimilates to the acceleration force. This resultant traction force $F$ will produce a counterproductive torque to the driving motor, which is represented by the following relationship:

$$
T_{L}=F \frac{r}{G},
$$

where $T_{L}$ is the torque produced by the driving motor, $r$ is the tyre radius of the $\mathrm{EV}$, and $G$ is the gearing ratio.

2.2. Motor Dynamics. The EV is driven by a DC motor and the dynamics of which are given by [11]

$$
\begin{aligned}
& J \frac{d \omega}{d t}=L_{a f} i^{2}-B \omega-T_{L}, \\
& \left(L_{a}+L_{\text {field }}\right) \frac{d i}{d t}=V-\left(R_{a}+R_{f}\right) i-L_{a f} i \omega,
\end{aligned}
$$

where $J$ is the inertia of the motor, including the gearing system and the tyres, $\omega$ is the motor angular speed, $i$ is the armature current (also field current), $L_{a}, R_{a}, L_{\text {field }}$, and $R_{f}$ are the armature inductance, armature resistance, field winding inductance, and field winding resistance, respectively, $B$ is the viscous coefficient, $T_{L}$ corresponds to the external torque, $V$ is the control input voltage, and $L_{a f}$ is the mutual inductance between the armature winding and the field winding, generally nonlinear because of saturation. In this paper the resistance in the armature winding $R_{a}$ of the DC motor is considered to be varying as the armature winding resistance of the DC motor changes as the temperature varies. 
TABle 1: Parameters of the EV system [11].

\begin{tabular}{lccc}
\hline & Motor & & Vehicle \\
\hline$L_{a}+L_{\text {field }}(\mathrm{mH})$ & 6.008 & $M(\mathrm{~kg})$ & 800 \\
$R_{a}+R_{f}(\Omega)$ & 0.12 & $A\left(\mathrm{~m}^{2}\right)$ & 1.8 \\
$B(\mathrm{~N} . \mathrm{M} . \mathrm{s})$. & 0.0002 & $\rho\left(\mathrm{kg} / \mathrm{m}^{3}\right)$ & 1.25 \\
$J\left(\mathrm{~kg} \mathrm{~m}^{2}\right)$ & 0.05 & $C_{d}$ & 0.3 \\
$L_{a f}(\mathrm{mH})$ & 1.766 & $\left(^{\circ}\right)$ & 0 \\
$V(\mathrm{~V})$ & $0 \sim 48$ & $\mu_{\mathrm{rr}}$ & 0.015 \\
$i(\mathrm{~A})$ & $78 \mathrm{~A}(250 \mathrm{max})$ & 0.25 \\
$\omega(\mathrm{r} / \mathrm{min})$ & $2800(v=25 \mathrm{~km} / \mathrm{h})$ & $G$ & 11 \\
\hline
\end{tabular}

$\Delta M=150(\mathrm{~kg})$ for $t \leq 195$ and $t \geq 780(\mathrm{~kg}), \Delta M=220$ for $t>195$, and $t \leq 585$ and $\Delta M=300(\mathrm{~kg})$ for $t>585$ and $t<780$.

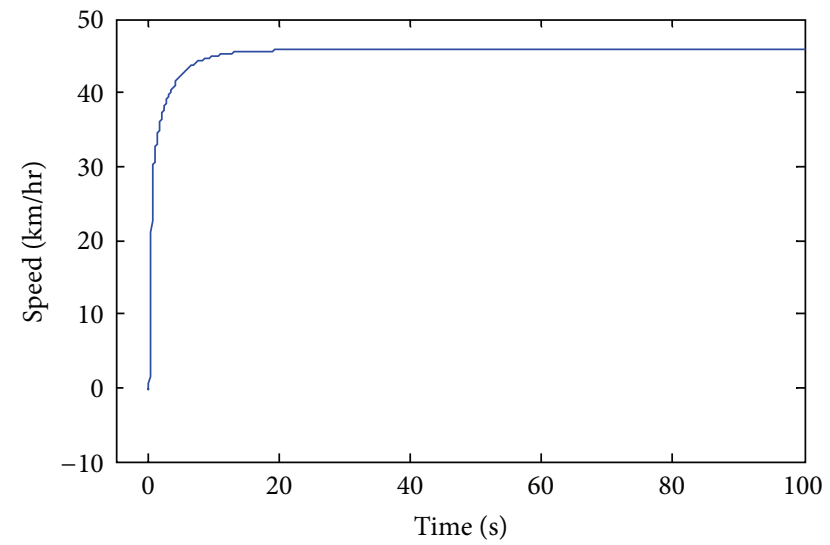

FIgURE 2: Open-loop response of the EV system.

2.3. Complete Dynamics and Open-Loop Response. With vehicle dynamics (1) and motor dynamics (3), the complete dynamics of the EV system becomes [11]

$$
\begin{aligned}
& \left\{J+m \frac{r^{2}}{G^{2}}\right\} \frac{d \omega}{d t}=L_{a f} i^{2}-B \omega \\
& -\frac{r}{G}\left\{\begin{array}{l}
\mu_{r r} m g \\
+\frac{1}{2} \rho A C_{d} v^{2}+m g \sin \phi
\end{array}\right\} \\
& \left(L_{a}+L_{\text {field }}\right) \frac{d i}{d t}=V-\left(R_{a}+R_{f}\right) i-L_{a f} i \omega .
\end{aligned}
$$

By using (4), the open-loop response of a light-weighted all-electric vehicle is studied. The parameters used on a light-weighted all-electric vehicle are specified in Table 1. The simulation result of open-loop response is shown in Figure 2. The plot shows the full power speed characteristics. As given in Table 1 the desired nominal speed is $v=25 \mathrm{~km} / \mathrm{hr}$ whereas in open-loop conditions the speed is beyond $40 \mathrm{~km} / \mathrm{hr}$ which is not acceptable. Therefore, it is required to design proper controller.

The relation between the driving velocity of the vehicle $v$ and the motor angular speed $\omega$ is given as

$$
v=\frac{r}{G} \omega
$$

where $r$ is the tyre radius of the $\mathrm{EV}$ and $G$ is the gearing ratio.

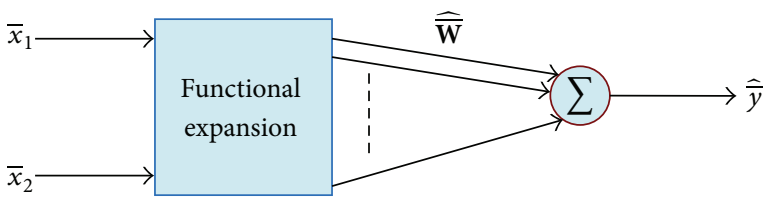

FIgURE 3: Structure of CNN.

2.4. Structure of Neural Network. In this paper, a single layer $\mathrm{CNN}$ is considered for the $\mathrm{NN}$ structure. CNN consists of a functional expansion (FE) block and a single-layer perceptron network. The purpose of the FE block is to increase the dimension of the input pattern so as to improve representation of the input pattern in a higher dimensional space. Chebyshev expansions are frequently used for approximations to functions as they are much more efficient than other power series expansion of the same degree. Among orthogonal polynomials, the Chebyshev polynomials which are derived from the solution of the Chebyshev differential equation occupy an important place, since, in the case of a broad class of functions, expansions in Chebyshev polynomials converge more rapidly than expansions in other set of polynomials. Hence, we consider the Chebyshev polynomials as basis functions for the neural network.

The Chebyshev polynomials can be generated by the following recursive formula [17]:

$$
T_{i+1}(\bar{x})=2 \bar{x} T_{i}(\bar{x})-T_{i-1}(\bar{x}), \quad T_{0}(\bar{x})=1,
$$

where $T_{i}(\bar{x})$ is a Chebyshev polynomial, $i$ is the order of Chebyshev polynomials chosen, and here $\bar{x}$ is scalar quantity. $T_{1}(\bar{x})$ can be chosen as $\bar{x}, 2 \bar{x}, 2 \bar{x}-1$ or $2 \bar{x}+1$. In this paper $T_{1}(\bar{x})$ is chosen as $\bar{x}$. For example, an enhanced pattern using the Chebyshev polynomials for $\bar{x} \in \Re^{2}$ is obtained as

$$
\bar{\phi}(\bar{x})=\left[\begin{array}{llllll}
1 & T_{1}\left(\bar{x}_{1}\right) & T_{2}\left(\bar{x}_{1}\right) \cdots & T_{1}\left(\bar{x}_{2}\right) & T_{2}\left(\bar{x}_{2}\right) \cdots
\end{array}\right]^{T},
$$

where $T_{i}\left(\bar{x}_{j}\right)$ is a Chebyshev polynomial, $i$ is the order of the selected Chebyshev polynomial, and $j=1,2 . \bar{\phi}(\bar{x})$ denotes the Chebyshev polynomial basis function.

Referring to Figure 3, the architecture of the CNN consists of two parts [17], namely, numerical transformation part and learning part. The numerical transformation is the FE 
of the input pattern consisting of a finite set of Chebyshev polynomials. Consequently, the Chebyshev polynomial basis can be considered as a new input vector. The learning part involves functional-link neural network based on Chebyshev polynomials. The CNN is a single-layered neural network, and, in general, its learning is fast $[16,17]$.

On the basis of approximation property of CNN [19], a general nonlinear function $\bar{y}(\bar{x})$ can be approximated by $\mathrm{CNN}$ as

$$
\bar{y}(\bar{x})=\overline{\mathbf{W}}^{T} \bar{\phi}+\bar{\varepsilon},
$$

where $\bar{\varepsilon}$ is the $\mathrm{CNN}$ functional reconstruction error vector and $\|\bar{\varepsilon}\| \leq \bar{\varepsilon}_{N}$ which is bounded, $\overline{\mathbf{W}}$ is the optimal weight matrix, and $\bar{\phi}$ denotes the Chebyshev polynomial basis function. The output of the $\mathrm{CNN}$ is given by

$$
\hat{\bar{y}}=\widehat{\overline{\mathbf{W}}}^{T} \bar{\phi}
$$

where $\widehat{\mathbf{W}}$ is the estimate of the optimal weight matrix $\overline{\mathbf{W}}$.

\section{Problem Statement}

The complete dynamics in (4) can be described as

$$
\begin{aligned}
\dot{\mathbf{X}} & =f(\mathbf{X})+g(\mathbf{X}) u, \\
y & =h(\mathbf{X}),
\end{aligned}
$$

where

$$
\begin{aligned}
& \mathbf{X}=\left[\begin{array}{l}
x_{1} \\
x_{2}
\end{array}\right]=\left[\begin{array}{c}
\omega \\
i
\end{array}\right], \\
& f(\mathbf{X})=\left[\frac { 1 } { J + m ( r ^ { 2 } / G ^ { 2 } ) } \left\{L_{a f} x_{2}{ }^{2}-B x_{1}-\frac{r}{G}\right.\right. \\
& \left.\times\left(\begin{array}{c}
\mu_{r r} m g+\frac{1}{2} \rho A C_{d} \frac{r^{2}}{G^{2}} x_{1}^{2} \\
+m g \sin \phi
\end{array}\right)\right\} \\
& \left.-\frac{R_{a}+R_{f}}{L_{a}+L_{\text {field }}} x_{2}-\frac{L_{a f}}{L_{a}+L_{\text {field }}} x_{1} x_{2}\right] \text {, } \\
& g(\mathbf{X})=\left[\begin{array}{c}
0 \\
\frac{1}{L_{a}+L_{\text {field }}}
\end{array}\right], \quad h(\mathbf{X})=x_{1} .
\end{aligned}
$$

Assuming the hill climbing angle $\phi$ to be zero, the nonlinear functions $f(\mathbf{X})$ and $g(\mathbf{X})$ become

$$
\begin{gathered}
f(\mathbf{X})=\left[\begin{array}{c}
\frac{K_{1}}{m+K_{2}}\left\{K_{3} x_{2}{ }^{2}-K_{4} x_{1}-K_{5} x_{1}{ }^{2}-K_{6} m\right\} \\
-K_{7} x_{2}-K_{8} x_{1} x_{2}
\end{array}\right], \\
g(\mathbf{X})=\left[\begin{array}{c}
0 \\
K_{9}
\end{array}\right], \quad h(\mathbf{X})=x_{1},
\end{gathered}
$$

where $K_{1}=G^{2} / r^{2}, K_{2}=\left(G^{2} / r^{2}\right) J, K_{3}=L_{a f}, K_{4}=B, K_{5}=$ $(1 / 2) \rho A C_{d}\left(r^{3} / G^{3}\right), K_{6}=(r / G) \mu_{r r} g, K_{7}=\left(R_{a}+R_{f}\right) /\left(L_{a}+\right.$ $\left.L_{\text {field }}\right), K_{8}=L_{a f} /\left(L_{a}+L_{\text {field }}\right)$, and $K_{9}=1 /\left(L_{a}+L_{\text {field }}\right)$.

As a result, the EV system becomes

$$
\begin{aligned}
& \dot{x}_{1}=\frac{K_{1}}{m+K_{2}}\left\{K_{3} x_{2}^{2}-K_{4} x_{1}-K_{5} x_{1}^{2}-K_{6} m\right\}, \\
& \dot{x}_{2}=-K_{7} x_{2}-K_{8} x_{1} x_{2}+K_{9} u, \\
& y=x_{1} .
\end{aligned}
$$

The aim of this paper is to compare and test the performance of CNN based backstepping controller with a CNN based optimal adaptive controller, which forces the plant output $y$ to track a specified reference trajectory $y_{d}$ in the presence of time varying mass " $m$ " and varying armature winding resistance $\left(R_{a}\right)$, aerodynamic drag coefficient $C_{d}$, and the rolling resistance coefficient $\mu_{r r}$; that is,

$$
\lim _{t \rightarrow \infty}\left(y-y_{d}\right)=0 \text {. }
$$

\section{Nonlinear Backstepping Controller Design}

The nonlinear backstepping controller is designed in the following ways.

4.1. Conventional Backstepping Controller. In this subsection, the steps involved in the development of backstepping controller for system (14) are discussed.

By selecting the following state transformation:

$$
\begin{aligned}
& z_{1}=x_{1}, \\
& z_{2}=\dot{x}_{1},
\end{aligned}
$$

the system (14) becomes

$$
\begin{aligned}
\dot{z}_{1}= & z_{2}, \\
\dot{z}_{2}= & -\frac{2 K_{1} K_{3} K_{7}}{m+K_{2}} x_{2}{ }^{2}-\frac{2 K_{1} K_{3} K_{8}}{m+K_{2}} z_{1} x_{2}{ }^{2}-\frac{K_{1} K_{4}}{m+K_{2}} z_{2} \\
& \quad-\frac{2 K_{1} K_{5}}{m+K_{2}} z_{1} z_{2}+\frac{2 K_{1} K_{3} K_{9}}{m+K_{2}} x_{2} u, \\
y= & z_{1},
\end{aligned}
$$

which is in the strict feedback form [24, 25]. In this case, all the nonlinear functions are considered known. The error dynamics is defined as

$$
\begin{aligned}
& e_{1}=z_{1}-z_{1 d}=z_{1}-y_{d} \\
& e_{2}=z_{2}-z_{2 d} .
\end{aligned}
$$

The time derivative of $e_{1}$ can be found as

$$
\dot{e}_{1}=\dot{z}_{1}-\dot{y}_{d} .
$$

From (17) and (19), we have (20) as

$$
\dot{e}_{1}=e_{2}+z_{2 d}-\dot{y}_{d}
$$


By selecting $z_{2 d}=\dot{y}_{d}-\bar{k}_{1} e_{1},(21)$ yields

$$
\dot{e}_{1}=e_{2}-\bar{k}_{1} e_{1},
$$

where $\bar{k}_{1}$ is the positive constant.

Differentiating (19),

$$
\dot{e}_{2}=\dot{z}_{2}-\dot{z}_{2 d}
$$

Substituting $\dot{z}_{2}$ from (17), (23) becomes

$$
\begin{aligned}
\dot{e}_{2}= & -\frac{2 K_{1} K_{3} K_{7}}{m+K_{2}} x_{2}^{2}-\frac{2 K_{1} K_{3} K_{8}}{m+K_{2}} z_{1} x_{2}^{2} \\
& -\frac{K_{1} K_{4}}{m+K_{2}} z_{2}-\frac{2 K_{1} K_{5}}{m+K_{2}} z_{1} z_{2} \\
& +\frac{2 K_{1} K_{3} K_{9}}{m+K_{2}} x_{2} u-\dot{z}_{2 d} .
\end{aligned}
$$

To stabilize (24), the control effort $u$ is chosen as

$$
\begin{aligned}
u=\left\{\begin{array}{l}
\dot{z}_{2 d}-e_{1}+\frac{2 K_{1} K_{3} K_{7}}{m+K_{2}} x_{2}{ }^{2}+\frac{2 K_{1} K_{3} K_{8}}{m+K_{2}} z_{1} x_{2}{ }^{2} \\
+\frac{K_{1} K_{4}}{m+K_{2}} z_{2}+\frac{2 K_{1} K_{5}}{m+K_{2}} z_{1} z_{2}-\bar{k}_{2} e_{2}
\end{array}\right\} \\
\times \frac{1}{\left\{\left(2 K_{1} K_{3} K_{9}\right) /\left(m+K_{2}\right)\right\} x_{2}},
\end{aligned}
$$

where $\bar{k}_{2}$ is the positive constant.

Substituting (25) in (24) gives

$$
\dot{e}_{2}=-e_{1}-\bar{k}_{2} e_{2}
$$

To prove the convergence of the EV system a Lyapunov function is chosen as

$$
L_{1}=\frac{1}{2} e_{1}^{2}+\frac{1}{2} e_{2}^{2}
$$

The time derivative of $(27)$ is

$$
\dot{L}_{1}=e_{1} \dot{e}_{1}+e_{2} \dot{e}_{2} \text {. }
$$

Using (22) and (26) we get

$$
\dot{L}_{1}=-\bar{k}_{1} e_{1}^{2}-\bar{k}_{2} e_{2}^{2}
$$

Thus, it can be easily seen that the system is globally asymptotically stable.

4.2. CNN Based Backstepping Controller. The EV system in (17) is rewritten in the following form for the implementation of the CNN based backstepping controller:

$$
\begin{aligned}
\dot{z}_{1} & =z_{2}, \\
\dot{z}_{2} & =f(x, z)+g(x, z) u, \\
y & =z_{1} .
\end{aligned}
$$

Here the nonlinear functions $f(x, z)$ and $g(x, z)$ are unknown as $m, R_{a}, C_{d}$, and $\mu_{r r}$ are varying with time.

The error dynamics is defined as

$$
\begin{aligned}
& e_{1}=z_{1}-z_{1 d}=z_{1}-y_{d} \\
& e_{2}=z_{2}-z_{2 d} .
\end{aligned}
$$

The CNN based backstepping controller is designed in the following two steps.

Step 1 . The time derivative of $e_{1}$ is given by

$$
\dot{e}_{1}=\dot{z}_{1}-\dot{y}_{d}
$$

Using (30) and (31), (32) becomes

$$
\dot{e}_{1}=e_{2}+z_{2 d}-\dot{y}_{d}
$$

Stabilize (33) by choosing

$$
z_{2 d}=\dot{y}_{d}-k_{1} e_{1} \text {, }
$$

where $k_{1}$ is the positive constant.

Substituting (34) in (33), $\dot{e}_{1}$ becomes

$$
\dot{e}_{1}=e_{2}-k_{1} e_{1} \text {. }
$$

Step 2. The time derivative of $e_{2}$ can be obtained as

$$
\dot{e}_{2}=\dot{z}_{2}-\dot{z}_{2 d}
$$

By using (30), (36) results in

$$
\dot{e}_{2}=f(x, z)+g(x, z) u-\dot{z}_{2 d} .
$$

The unknown nonlinear functions $f(x, z)$ and $g(x, z)$ will be estimated by CNN 1 and CNN 2, respectively. The nonlinear functions $f(x, z)$ and $g(x, z)$ can be represented by a CNN as

$$
\begin{aligned}
& f=\mathbf{W}_{1}^{T} \phi_{1}+\varepsilon_{1}, \\
& g=\mathbf{W}_{2}^{T} \phi_{2}+\varepsilon_{2},
\end{aligned}
$$

where $\varepsilon_{1}$ and $\varepsilon_{2}$ are the bounded $\mathrm{CNN}$ approximation errors, $\mathbf{W}_{1}$ and $\mathbf{W}_{2}$ are the optimal weight matrices, and $\phi_{1}$ and $\phi_{2}$ are the basis functions.

The estimate $\widehat{f}$ of $f$ and $\widehat{g}$ of $g$ can be written as

$$
\begin{gathered}
\widehat{f}=\widehat{\mathbf{W}}_{1}^{T} \phi_{1}, \\
\widehat{g}=\widehat{\mathbf{W}}_{2}^{T} \boldsymbol{\phi}_{2},
\end{gathered}
$$

where $\widehat{\mathbf{W}}_{1}$ is the estimate of the $\mathbf{W}_{1}$ and $\widehat{\mathbf{W}}_{2}$ is the estimate of the $\mathbf{W}_{2}$. have

Adding and subtracting $\widehat{g}(x, z) u$ and rearranging, (37) we

$$
\dot{e}_{2}=f(x, z)+\{g(x, z)-\widehat{g}(x, z)\} u-\dot{z}_{2 d}+\widehat{g}(x, z) u \text {. }
$$




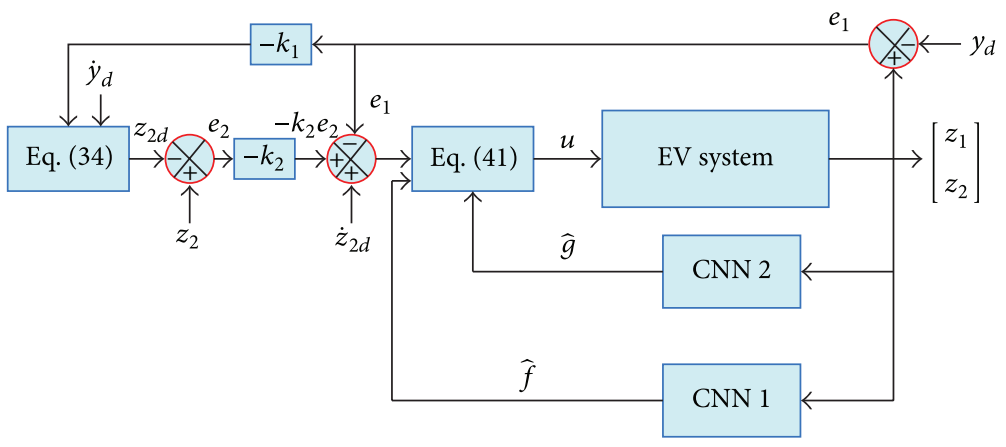

FIGURE 4: Block diagram of CNN based backstepping controller.

To stabilize (40), the control effort $u$ is chosen as

$$
u=\frac{1}{\widehat{g}(x, z)}\left\{-\widehat{f}(x, z)+\dot{z}_{2 d}-k_{2} e_{2}-e_{1}\right\},
$$

where $k_{2}$ is the positive constant. The block diagram of the overall system is presented in Figure 4.

Substituting (41) in (40), $\dot{e}_{2}$ becomes

$$
\dot{e}_{2}=\{f(x, z)-\widehat{f}(x, z)\}+\{g(x, z)-\widehat{g}(x, z)\} u-k_{2} e_{2}-e_{1} .
$$

Define the estimation error as

$$
\begin{aligned}
& \tilde{f}=f-\widehat{f}, \\
& \tilde{g}=g-\widehat{g} .
\end{aligned}
$$

Using (38) and (39) in (43) gives

$$
\begin{gathered}
\tilde{f}=\widetilde{\mathbf{W}}_{1}^{T} \phi_{1}+\varepsilon_{1}, \\
\tilde{g}=\widetilde{\mathbf{W}}_{2}^{T} \phi_{2}+\varepsilon_{2},
\end{gathered}
$$

where $\widetilde{\mathbf{W}}_{1}=\mathbf{W}_{1}-\widehat{\mathbf{W}}_{1}$ and $\widetilde{\mathbf{W}}_{2}=\mathbf{W}_{2}-\widehat{\mathbf{W}}_{2}$ are weight errors.

Now by applying (44) in (42) $\dot{e}_{2}$ becomes

$$
\dot{e}_{2}=\widetilde{\mathbf{W}}_{1}^{T} \phi_{1}+\varepsilon_{1}+\left(\widetilde{\mathbf{W}}_{2}^{T} \phi_{2}+\varepsilon_{2}\right) u-k_{2} e_{2}-e_{1} .
$$

Two standard assumptions, which are commonly used in the neural networks literature, are given below [26].

Assumption 1. The optimal weights $\mathbf{W}_{1}$ and $\mathbf{W}_{2}$ are bounded by known positive values so that

$$
\left\|\mathbf{W}_{1}\right\|_{F} \leq \mathbf{W}_{1 M}, \quad\left\|\mathbf{W}_{2}\right\|_{F} \leq W_{2 M} .
$$

We only need to know that ideal weights exist to prove the convergence analysis. The exact value of the ideal weights need not be known. The symbol $\|\bullet\|_{F}$ denotes the Frobenius norm; that is, given a matrix $\mathbf{A}$, the Frobenius norm is defined by

$$
\|\mathbf{A}\|_{F}^{2}=\operatorname{tr}\left(\mathbf{A}^{T} \mathbf{A}\right)=\sum_{i, j} a_{i j}^{2} .
$$

Assumption 2. The desired trajectory $y_{d}$ and its derivatives up to second order are bounded.

Based on the above Assumptions 1 and 2, the stability analysis is given in Section 4.3.

\subsection{Stability Analysis}

Theorem 3. Consider the EV system (30) and control input (41) satisfying Assumptions 1 and 2. If the weights of the CNN 1 and CNN 2 are updated according to adaptation law given in (48) and (49), respectively,

$$
\begin{aligned}
& \dot{\widehat{\mathbf{W}}}_{1}=\eta_{1} \phi_{1} e_{2}-\bar{\rho} \eta_{1}\left\|e_{2}\right\| \widehat{\mathbf{W}}_{1}, \\
& \dot{\widehat{\mathbf{W}}}_{2}=\eta_{2} \phi_{2} u e_{2}-\bar{\rho} \eta_{2}\left\|e_{2}\right\||u| \widehat{\mathbf{W}}_{2},
\end{aligned}
$$

where $\eta_{1}$ and $\eta_{2}$ are the learning rate and $\bar{\rho}$ is damping coefficient, then the weight errors $\widetilde{\mathbf{W}}_{1}=\mathbf{W}_{1}-\widehat{\mathbf{W}}_{1}, \widetilde{\mathbf{W}}_{2}=$ $\mathbf{W}_{2}-\widehat{\mathbf{W}}_{2}$ and the errors $e_{1}$ and $e_{2}$ are uniformly ultimately bounded (UUB).

Proof. Consider the Lyapunov function

$$
L_{2}=\frac{1}{2} e_{1}^{2}+\frac{1}{2} e_{2}^{2}+\frac{1}{2} \operatorname{tr}\left\{\widetilde{\mathbf{W}}_{1}^{T} \eta_{1}^{-1} \widetilde{\mathbf{W}}_{1}\right\}+\frac{1}{2} \operatorname{tr}\left\{\widetilde{\mathbf{W}}_{2}^{T} \eta_{2}^{-1} \widetilde{\mathbf{W}}_{2}\right\} .
$$

The time derivative of (50) is

$$
\begin{aligned}
\dot{L}_{2}= & e_{1} \dot{e}_{1}+e_{2} \dot{e}_{2}+\operatorname{tr}\left\{\widetilde{\mathbf{W}}_{1}^{T} \eta_{1}^{-1} \dot{\widetilde{\mathbf{W}}}_{1}\right\} \\
& +\operatorname{tr}\left\{\widetilde{\mathbf{W}}_{2}^{T} \eta_{2}^{-1} \dot{\widehat{\mathbf{W}}}_{2}\right\} .
\end{aligned}
$$

Now substitute $\dot{e}_{1}$ and $\dot{e}_{2}$ from (35) and (45), respectively, and perform a simple manipulation (i.e., using $x^{T} y=\operatorname{tr}\left\{x^{T} y\right\}=$ $\operatorname{tr}\left\{y x^{T}\right\}$, for placing weight matrices inside a trace operator). Then we have

$$
\begin{aligned}
\dot{L}_{2}= & -k_{1} e_{1}^{2}+e_{2}\left(\varepsilon_{1}+\varepsilon_{2} u-k_{2} e_{2}\right) \\
& +\operatorname{tr}\left\{\widetilde{\mathbf{W}}_{1}^{T}\left(\phi_{1} e_{2}+\eta_{1}^{-1} \dot{\widetilde{\mathbf{W}}}_{1}\right)\right\} \\
& +\operatorname{tr}\left\{\widetilde{\mathbf{W}}_{2}^{T}\left(\phi_{2} e_{2}+\eta_{2}^{-1} \dot{\widetilde{\mathbf{W}}}_{2}\right)\right\} .
\end{aligned}
$$

With the adaptation law given in (48) and (49), (52) becomes

$$
\begin{aligned}
\dot{L}_{2}= & -k_{1} e_{1}^{2}+e_{2}\left(\varepsilon_{1}+\varepsilon_{2} u-k_{2} e_{2}\right) \\
& +\operatorname{tr}\left\{\widetilde{\mathbf{W}}_{1}^{T}\left(\bar{\rho}\left\|e_{2}\right\| \widehat{\mathbf{W}}_{1}\right)\right\} \\
& +\operatorname{tr}\left\{\widetilde{\mathbf{W}}_{2}^{T}\left(\bar{\rho}\left\|e_{2}\right\||u| \widehat{\mathbf{W}}_{2}\right)\right\} .
\end{aligned}
$$


Apply the following inequality [27]:

$$
\begin{aligned}
\operatorname{tr}\left[\widetilde{\mathbf{W}}^{T}(\mathbf{W}-\widetilde{\mathbf{W}})\right] & =\langle\widetilde{\mathbf{W}}, \mathbf{W}\rangle_{F}-\|\widetilde{\mathbf{W}}\|_{F}^{2} \\
& \leq\|\widetilde{\mathbf{W}}\|_{F} \mathbf{W}_{M}-\|\widetilde{\mathbf{W}}\|_{F}^{2} .
\end{aligned}
$$

And assume that the upper bounds are as follows:

$$
\left\|\varepsilon_{1}\right\| \leq \varepsilon_{1 M}, \quad\left\|\varepsilon_{2}\right\| \leq \varepsilon_{2 M}, \quad|u| \leq u_{S} .
$$

Now we can express (53) as

$$
\begin{aligned}
\dot{L}_{2} \leq & -k_{1}\left\|e_{1}\right\|^{2}+\left\|e_{2}\right\|\left(\varepsilon_{1 M}+\varepsilon_{2 M} u_{S}-k_{2}\left\|e_{2}\right\|\right) \\
& +\bar{\rho}\left\|e_{2}\right\|\left\{\left(\left\|\widetilde{\mathbf{W}}_{1}\right\|_{F} W_{1 M}-\left\|\widetilde{\mathbf{W}}_{1}\right\|_{F}^{2}\right)\right\} \\
& +\bar{\rho}\left\|e_{2}\right\| u_{S}\left\{\left(\left\|\widetilde{\mathbf{W}}_{2}\right\|_{F} W_{2 M}-\left\|\widetilde{\mathbf{W}}_{2}\right\|_{F}^{2}\right)\right\} .
\end{aligned}
$$

As the first term is always negative, now defining nonnegative term and completing the square terms in (56) yield

$$
\begin{aligned}
\dot{L}_{2} \leq-\left\|e_{2}\right\|\left[k_{2}\right. & \left\|e_{2}\right\|-\varepsilon_{1 M}-\varepsilon_{2 M} u_{S} \\
& +\bar{\rho}\left(\left\|\widetilde{\mathbf{W}}_{1}\right\|_{F}^{2}-\frac{W_{1 M}}{2}\right)^{2} \\
& -\bar{\rho} \frac{W_{1 M}^{2}}{4}+\bar{\rho} u_{S}\left(\left\|\widetilde{\mathbf{W}}_{2}\right\|_{F}^{2}-\frac{W_{2 M}}{2}\right)^{2} \\
& \left.-\bar{\rho} u_{S} \frac{W_{2 M}^{2}}{4}\right]
\end{aligned}
$$

which is negative as long as either (58), (59), or (60) holds

$$
\begin{gathered}
\frac{\left[\varepsilon_{1 M}+\varepsilon_{2 M} u_{S}+\bar{\rho}\left(W_{1 M}^{2} / 4+u_{S} W_{2 M}^{2} / 4\right)\right]}{k_{2}}<\left\|e_{2}\right\|, \\
\frac{W_{1 M}}{2}+\sqrt{\left(\frac{W_{1 M}^{2}}{4}\right)+\frac{\varepsilon_{1 M}}{\bar{\rho}}} \leq\left\|\widetilde{\mathbf{W}}_{1}\right\|_{F} .
\end{gathered}
$$

Or

$$
\frac{W_{2 M}}{2}+\sqrt{u_{S}\left(\frac{W_{2 M}^{2}}{4}+\frac{\varepsilon_{2 M}}{\bar{\rho}}\right)} \leq\left\|\widetilde{\mathbf{W}}_{2}\right\|_{F} .
$$

Thus, $\dot{L}_{2}$ is negative outside a compact set. According to a standard Lyapunov theorem extension [28], this demonstrates uniform ultimate boundedness of weight errors $\widetilde{\mathbf{W}}_{1}$, $\widetilde{\mathbf{W}}_{2}$ and errors $e_{1}, e_{2}$.

\section{Nonlinear Optimal Controller Design Using CNN}

The following approach is used to design the nonlinear optimal controller for the EV system (17).

The tracking errors are defined as

$$
\begin{aligned}
& e(t)=z_{1}(t)-y_{d}(t), \\
& \dot{e}(t)=\dot{z}_{1}(t)-\dot{y}_{d}(t)=z_{2}(t)-\dot{y}_{d}(t) .
\end{aligned}
$$

And the filtered tracking error is defined as

$$
r(t)=\dot{e}(t)+\Lambda e(t),
$$

where $\Lambda$ is the positive constant.

Differentiating (62) and rearranging it, we have

$$
\dot{r}(t)=\Lambda r(t)-\ddot{y}_{d}(t)+\dot{z}_{2}(t)-\Lambda^{2} e(t) .
$$

Substituting $\dot{z}_{2}(t)$ from EV system (17), (63) becomes

$$
\dot{r}(t)=\Lambda r(t)-\ddot{y}_{d}(t)+X_{1 x}+F_{1 x} u-\Lambda^{2} e(t),
$$

where $X_{1 x}=-\left(2 K_{1} K_{3} K_{7} /\left(m+K_{2}\right)\right) x_{2}{ }^{2}-\left(2 K_{1} K_{3} K_{8} /(m+\right.$ $\left.\left.K_{2}\right)\right) z_{1} x_{2}{ }^{2}-\left(\left(K_{1} K_{4}\right) /\left(m+K_{2}\right)\right) z_{2}-\left(\left(2 K_{1} K_{5}\right) /\left(m+K_{2}\right)\right) z_{1} z_{2}$ and $F_{1 x}=\left(\left(2 K_{1} K_{3} K_{9}\right) /\left(m+K_{2}\right)\right) x_{2}$.

System (64) may be written as

$$
\dot{r}(t)=\Lambda r(t)+F_{1 x} u(t)+h(x),
$$

where

$$
h(x)=X_{1 x}-\ddot{y}_{d}(t)-\Lambda^{2} e(t) .
$$

Now we define an auxiliary control input $\bar{u}(t)$, which is to be optimized in the next subsection as

$$
\bar{u}(t)=h(x)+F_{1 x} u(t)
$$

with $u(t)$ as the control input. The closed-loop system becomes

$$
\dot{r}(t)=\Lambda r(t)+\bar{u}(t) .
$$

5.1. Optimal Controller Using Hamilton-Jacobi-Bellman ( $H$ $J$-B) Optimization. The augmented system [27] is achieved using (62) and (68)

$$
\left[\begin{array}{l}
\dot{e} \\
\dot{r}
\end{array}\right]=\left[\begin{array}{cc}
-\Lambda & I \\
0 & \Lambda
\end{array}\right]\left[\begin{array}{l}
e \\
r
\end{array}\right]+\left[\begin{array}{l}
0 \\
I
\end{array}\right] \bar{u}(t)
$$

or with shorter notation

$$
\dot{\widetilde{\mathbf{z}}}(t)=\mathbf{A} \widetilde{\mathbf{z}}(t)+\mathbf{B} \bar{u}(t)
$$

with $\widetilde{\mathbf{z}}(t)$ being defined as $\widetilde{\mathbf{z}}(t)=\left[\begin{array}{ll}e(t) & r(t)\end{array}\right]^{T}, \mathbf{A}=\left[\begin{array}{ll}-\Lambda & I ;\end{array}\right.$ $\left.\begin{array}{ll}0 & \Lambda\end{array}\right]$, and $\mathbf{B}=\left[\begin{array}{ll}0 & I\end{array}\right]^{T}$. A quadratic performance measure $J(\bar{u})$ is as follows:

$$
J(\bar{u})=\int_{t_{0}}^{\infty} L(\widetilde{\mathbf{z}}, \bar{u}) d t
$$

with the Lagrangian

$$
\begin{aligned}
L(\widetilde{\mathbf{z}}, \bar{u}) & =\frac{1}{2} \widetilde{\mathbf{z}}^{T}(t) \mathbf{Q} \widetilde{\mathbf{z}}(t)+\frac{1}{2} \bar{u}^{T}(t) R \bar{u}(t) \\
& =\frac{1}{2}\left[\begin{array}{ll}
e & r
\end{array}\right]\left[\begin{array}{ll}
Q_{11} & Q_{12} \\
Q_{12}^{T} & Q_{22}
\end{array}\right]\left[\begin{array}{l}
e \\
r
\end{array}\right]+\frac{1}{2} \bar{u}^{T} R \bar{u} .
\end{aligned}
$$

The objective is to find the auxiliary control input $\bar{u}(t)$ that minimizes the quadratic performance measure $J(\bar{u})$ subject 
to the constraints imposed by (69) which will be denoted by $\bar{u}^{*}(t)$ in the presence of known nonlinearities.

A necessary and sufficient condition for $\bar{u}^{*}(t)$ is that there exist a function $V=V(\widetilde{\mathbf{z}}, t)$, which is accredited as the value function and satisfies the H-J-B equation [29]

$$
\frac{\partial V(\widetilde{\mathbf{z}}, t)}{\partial t}+\min _{\bar{u}}\left[H\left(\widetilde{\mathbf{z}}, \bar{u}, \frac{\partial V(\widetilde{\mathbf{z}}, t)}{\partial t}, t\right)\right]=0
$$

where the Hamiltonian of optimization is described as

$$
H\left(\widetilde{\mathbf{z}}, \bar{u}, \frac{\partial V(\widetilde{\mathbf{z}}, t)}{\partial t}, t\right)=L(\widetilde{\mathbf{z}}, \bar{u})+\frac{\partial V(\widetilde{\mathbf{z}}, t)}{\partial t} \dot{\widetilde{\mathbf{z}}}
$$

and $V(\widetilde{\mathbf{z}}, t)$ satisfies the partial differential equation

$$
-\frac{\partial V(\widetilde{\mathbf{z}}, t)}{\partial t}=L\left(\widetilde{\mathbf{z}}, \bar{u}^{*}\right)+\frac{\partial V(\widetilde{\mathbf{z}}, t)}{\partial t} \dot{\tilde{\mathbf{z}}}
$$

The minimum is achieved for $\bar{u}(t)=\bar{u}^{*}(t)$, and the Hamiltonian is then given by

$$
\begin{aligned}
H^{*} & =\min _{\bar{u}}\left[L(\widetilde{\mathbf{z}}, \bar{u})+\frac{\partial V(\widetilde{\mathbf{z}}, t)}{\partial t} \dot{\tilde{\mathbf{z}}}\right] \\
& =H\left(\widetilde{\mathbf{z}}, \bar{u}^{*}, \frac{\partial V(\widetilde{\mathbf{z}}, t)}{\partial t}, t\right) \\
& =-\frac{\partial V(\widetilde{\mathbf{z}}, t)}{\partial t} .
\end{aligned}
$$

Lemma 4 (see [27]). The function $V$ composed of $\widetilde{\mathbf{z}}$ and $K$ satisfies the H-J-B equation

$$
V=\frac{1}{2} \widetilde{\mathbf{z}}^{T} \mathbf{P} \widetilde{\mathbf{z}}=\frac{1}{2} \widetilde{\mathbf{z}}^{T}\left[\begin{array}{cc}
K & 0 \\
0 & I
\end{array}\right] \widetilde{\mathbf{z}}
$$

where $\Lambda$ and $K$ in (62) and (77) respectively can be found from the Riccati differential equation

$$
\mathbf{P A}+\mathbf{A}^{T} \mathbf{P}^{T}-\mathbf{P B} R^{-1} \mathbf{B}^{T} \mathbf{P}+\dot{\mathbf{P}}+\mathbf{Q}=0 .
$$

The optimal control $\bar{u}^{*}(t)$ that minimizes (71) subject to (70) is

$$
\bar{u}^{*}(t)=-R^{-1} \mathbf{B}^{T} \mathbf{P} \widetilde{\mathbf{z}}=-R^{-1} r(t) .
$$

Let $\mathbf{Q}, R$ be chosen such that

$$
\mathbf{Q}=\left[\begin{array}{ll}
Q_{11} & Q_{12} \\
Q_{12}^{T} & Q_{22}
\end{array}\right]>0, \quad R^{-1}=Q_{22}
$$

with $Q_{12}+Q_{12}^{T}<0$ [27]. Then the $\Lambda$ and $K$ required in Lemma 4 can be calculated as given below

$$
\begin{gathered}
K=-\frac{1}{2}\left(Q_{12}+Q_{12}^{T}\right)>0, \\
\Lambda^{T} K+K \Lambda=Q_{11}
\end{gathered}
$$

with (82) solved for $\Lambda$.

Using (67) and (79), the input to the EV system is given as

$$
u^{*}(t)=\left(\operatorname{in} v\left(F_{x 1}\right)\right)\left\{\bar{u}^{*}(t)-h(x)\right\},
$$

where $h(x)$ is given by (66) and is assumed to be known. The following subsection details the stability analysis.

5.2. Stability Analysis. Suppose that $K$ and $\Lambda$ exist that satisfy Lemma 4 , and in addition, there exist constants $k_{1}^{\prime}$ and $k_{2}^{\prime}$ such that $0<k_{1}^{\prime}<k_{2}^{\prime}<\infty$, and the spectrum of $P$ is bounded in the sense that $k_{1}^{\prime} I<\mathbf{P}<k_{2}^{\prime} I$ on $\left(t_{0}, \infty\right)$. Then using the feedback control (79) into (70) results in the controlled nonlinear system becomes

$$
\dot{\widetilde{\mathbf{Z}}}(t)=\left\{\mathbf{A}-\mathbf{B} R^{-1} \mathbf{B}^{T} \mathbf{P}\right\} \widetilde{\mathbf{z}}(t) .
$$

The function $V(\widetilde{\mathbf{z}}, t)$ is chosen as a suitable Lyapunov function; it shows that $d V / d t<0$ for all $\|\widetilde{\mathbf{z}}\| \neq 0$. The time derivative of $V(\widetilde{\mathbf{z}}, t)$ is given by

$$
\frac{d V(\widetilde{\mathbf{z}}, t)}{d t}=\frac{\partial V(\widetilde{\mathbf{z}}, t)}{\partial t}+\frac{\partial V(\widetilde{\mathbf{z}}, t)}{\partial t} \dot{\widetilde{\mathbf{z}}}
$$

From the solution of the H-J-B equation (75) and using (85), it results that

$$
\frac{d V(\widetilde{\mathbf{z}}, t)}{d t}=-L\left(\widetilde{\mathbf{z}}, \bar{u}^{*}\right) .
$$

Substituting optimal control law (79) into (72), the time derivative of $V(\widetilde{\mathbf{z}}, t)$ becomes

$$
\begin{aligned}
\frac{d V(\widetilde{\mathbf{z}}, t)}{d t} & =-\frac{1}{2}\left\{\widetilde{\mathbf{z}}^{T} \mathbf{Q} \widetilde{\mathbf{z}}+\left(\mathbf{B}^{T} \mathbf{P} \widetilde{\mathbf{z}}\right)^{T} R^{-1}\left(\mathbf{B}^{T} \mathbf{P} \widetilde{\mathbf{z}}\right)\right\} \\
& <0 \text { just } \quad \forall t>0, \widetilde{\mathbf{z}} \neq 0 .
\end{aligned}
$$

The time derivative of the Lyapunov function is negative definite, implying global exponential stability.

5.3. CNN Based Optimal Adaptive Controller. In Section 5.1, the nonlinear function (66) is assumed to be known. This assumption is relaxed and $h(x)$ is treated as an unknown nonlinear function. The function $h(x)$ is estimated using CNN neural network. The nonlinear function $h(x)$ can be represented by a $\mathrm{CNN}$ as

$$
h(x)=\mathbf{W}^{T} \boldsymbol{\varphi}(x)+\varepsilon(x) \quad\|\varepsilon(x)\| \leq \varepsilon_{M},
$$




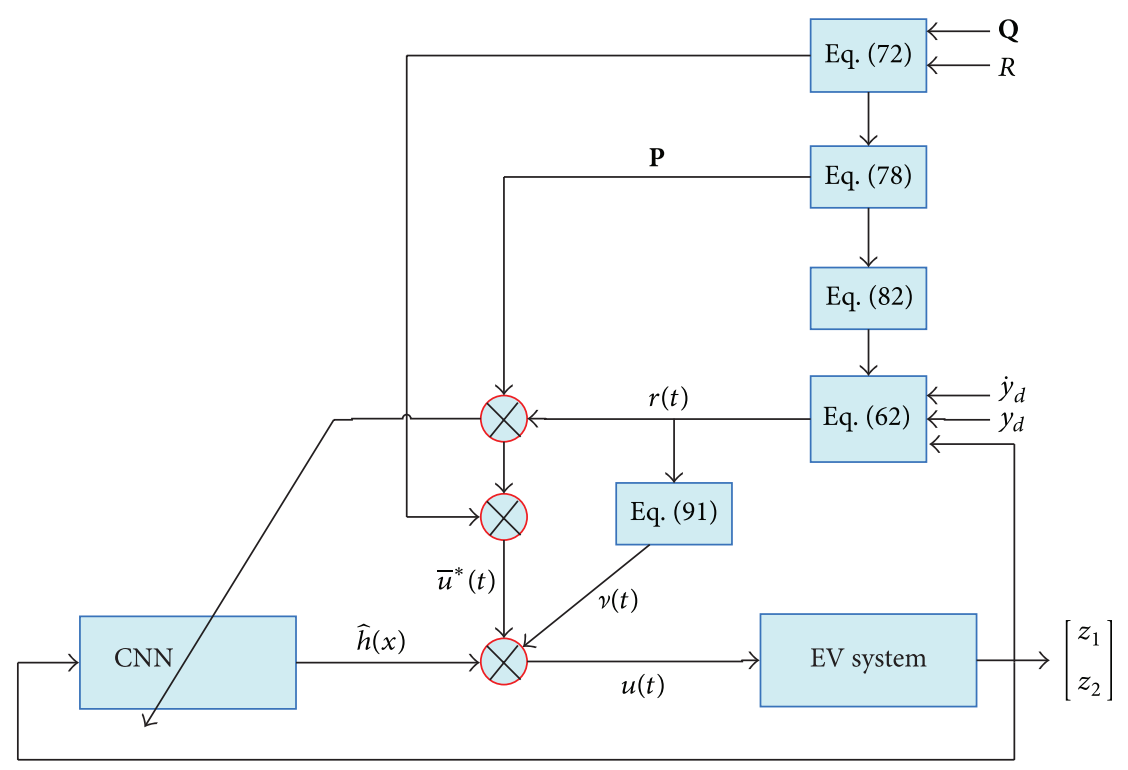

FIGURE 5: Block diagram of CNN based optimal adaptive controller.

where $\varphi(x)$ is a basis function for the CNN. The block diagram in Figure 5 demonstrates the CNN neural controller based on H-J-B optimization.

The estimate $\widehat{h}(x)$ of $h(x)$ can be written as

$$
\widehat{h}(x)=\widehat{\mathbf{W}}^{T} \varphi(x) .
$$

Using (67), (79), and (89), the input is given by

$$
u(t)=\left(\operatorname{in} v\left(F_{x 1}\right)\right)\left\{\bar{u}^{*}(t)-\widehat{\mathbf{W}}^{T} \varphi(x)-v(t)\right\},
$$

where $v(t)$ is a robustifying term, which is given by

$$
\nu(t)=\frac{-k_{z} r(t)}{\|r(t)\|}
$$

with $k_{z} \leq b_{d}$ and $r(t)$ being defined as the filtered tracking error in (62). Using (88) and (90), (65) becomes

$$
\dot{r}(t)=\Lambda r(t)+\bar{u}^{*}(t)+\widetilde{\mathbf{W}}^{T} \boldsymbol{\varphi}(x)+\varepsilon(x)-\nu(t),
$$

where $\widetilde{\mathbf{W}}=\mathbf{W}-\widehat{\mathbf{W}}$ is the weight-estimation error. Using (92) in (70) yields

$$
\dot{\tilde{\mathbf{z}}}(t)=\mathbf{A} \widetilde{\mathbf{z}}(t)+\mathbf{B}\left[\bar{u}^{*}(t)+\widetilde{\mathbf{W}}^{T} \boldsymbol{\varphi}(x)+\varepsilon(x)-v(t)\right]
$$

with A, B, and $\widetilde{\mathbf{z}}$ being given in (69) and (70).

Using the optimal control law (79) into (93), we have

$$
\dot{\tilde{\mathbf{z}}}(t)=\left(\mathbf{A}-\mathbf{B} R^{-1} \mathbf{B}^{T} \mathbf{P}\right) \widetilde{\mathbf{z}}(t)+\mathbf{B}\left\{\widetilde{\mathbf{W}}^{T} \boldsymbol{\varphi}(x)+\varepsilon(x)-v(t)\right\} .
$$

Theorem 5. Suppose the optimal control law $\bar{u}^{*}(t)$ given by (79) minimizes the quadratic performance measure $J(\bar{u})$ given in (71). If the weights of the CNN are updated according to adaptive learning law given by

$$
\dot{\widehat{\mathbf{W}}}=\boldsymbol{\varphi}(x) \widetilde{\mathbf{z}}^{T} \mathbf{P B} \Gamma-k\|\widetilde{\mathbf{z}}\| \widehat{\mathbf{W}}
$$

with $\Gamma>0$ and $k>0$, then the errors $e(t), r(t)$, and $\widetilde{\mathbf{W}}(t)$ are uniformly ultimately bounded.

Proof. Consider the following Lyapunov function:

$$
L_{3}=\frac{1}{2} \widetilde{\mathbf{z}}^{T}\left[\begin{array}{rr}
K & 0 \\
0 & 1
\end{array}\right] \widetilde{\mathbf{z}}+\frac{1}{2} \operatorname{tr}\left(\widetilde{\mathbf{W}}^{T} \Gamma^{-1} \widetilde{\mathbf{W}}\right),
$$

where $K$ is positive given by (81). The time derivative $\dot{L}_{3}$ of (96) becomes

$$
\dot{L}_{3}=\widetilde{\mathbf{z}}^{T} \mathbf{P} \dot{\overline{\mathbf{z}}}+\frac{1}{2} \widetilde{\mathbf{z}}^{T} \dot{\mathbf{P}} \widetilde{\mathbf{z}}+\operatorname{tr}\left(\widetilde{\mathbf{W}}^{T} \Gamma^{-1} \dot{\widetilde{\mathbf{W}}}\right) .
$$

Substituting $\dot{\tilde{z}}$ from (94), (97) becomes

$$
\begin{aligned}
\dot{L}_{3}= & \widetilde{\mathbf{z}}^{T} \mathbf{P A} \widetilde{\mathbf{z}}-\widetilde{\mathbf{z}}^{T} \mathbf{P B} R^{-1} \mathbf{B}^{T} \mathbf{P} \widetilde{\mathbf{z}}+\frac{1}{2} \widetilde{\mathbf{z}}^{T} \dot{\mathbf{P}} \widetilde{\mathbf{z}} \\
& +\widetilde{\mathbf{z}}^{T} \mathbf{P B}\left\{\widetilde{\mathbf{W}}^{T} \boldsymbol{\varphi}(x)+\varepsilon(x)-v(t)\right\} \\
& +\operatorname{tr}\left(\widetilde{\mathbf{W}}^{T} \Gamma^{-1} \dot{\overline{\mathbf{W}}}\right) .
\end{aligned}
$$

Using $\widetilde{\mathbf{z}}^{T} \mathbf{P A} \widetilde{\mathbf{z}}=(1 / 2) \widetilde{\mathbf{z}}^{T}\left\{\mathbf{A}^{T} \mathbf{P}+\mathbf{P A}\right\} \widetilde{\mathbf{z}}$ and from the Riccati equation (78), we obtain

$$
\frac{1}{2} \mathbf{A}^{T} \mathbf{P}+\frac{1}{2} \mathbf{P A}+\frac{1}{2} \dot{\mathbf{P}}=-\frac{1}{2} \mathbf{Q}+\frac{1}{2} \mathbf{P B} R^{-1} \mathbf{B}^{T} \mathbf{P} .
$$

Then applying (99) in (98) and performing a simple manipulation for placing weight matrices inside a trace operator, we have

$$
\begin{aligned}
\dot{L}_{3}= & -\frac{1}{2} \widetilde{\mathbf{z}}^{T} \mathbf{Q} \widetilde{\mathbf{z}}-\frac{1}{2} \widetilde{\mathbf{z}}^{T} \mathbf{P B} R^{-1} \mathbf{B}^{T} \mathbf{P} \widetilde{\mathbf{z}} \\
& +\widetilde{\mathbf{z}}^{T} \mathbf{P B}\{\varepsilon(x)-\nu(t)\} \\
& +\operatorname{tr}\left\{\widetilde{\mathbf{W}}^{T}\left(\Gamma^{-1} \dot{\overline{\mathbf{W}}}+\boldsymbol{\varphi} \widetilde{\mathbf{z}}^{T} \mathbf{P B}\right)\right\} .
\end{aligned}
$$


Now substitute the robustifying term (91), the adaptive learning law (95), and the following inequality:

$$
\begin{aligned}
\operatorname{tr}\left[\widetilde{\mathbf{W}}^{T}(\mathbf{W}-\widetilde{\mathbf{W}})\right] & =\langle\widetilde{\mathbf{W}}, \mathbf{W}\rangle_{F}-\|\widetilde{\mathbf{W}}\|_{F}^{2} \\
& \leq\|\widetilde{\mathbf{W}}\|_{F} W_{M}-\|\widetilde{\mathbf{W}}\|_{F}^{2}
\end{aligned}
$$

The time derivative $\dot{L}_{3}$ becomes

$$
\begin{aligned}
\dot{L}_{3} \leq & -\frac{1}{2}\|\widetilde{\mathbf{z}}\|^{2}\left\{\lambda_{\min }(\mathbf{Q})+\lambda_{\min }\left(R^{-1}\right)\right\} \\
& +\|\widetilde{\mathbf{z}}\| \varepsilon_{M}+k\|\widetilde{\mathbf{z}}\|\left(\|\widetilde{\mathbf{W}}\|_{F} W_{M}-\|\widetilde{\mathbf{W}}\|_{F}^{2}\right) .
\end{aligned}
$$

Completing the square terms yields

$$
\begin{aligned}
\dot{L}_{3} \leq-\frac{1}{2}\|\widetilde{\mathbf{z}}\|[ & \|\widetilde{\mathbf{z}}\|\left\{\lambda_{\min }(\mathbf{Q})+\lambda_{\min }\left(R^{-1}\right)\right\} \\
& \left.+k\left(\|\widetilde{\mathbf{W}}\|_{F}-\frac{1}{2} W_{M}\right)^{2}-\varepsilon_{M}-\frac{1}{4} k W_{M}^{2}\right]
\end{aligned}
$$

which is guaranteed to be negative as long as either (104) or (105) holds

$$
\begin{gathered}
\frac{\left(\varepsilon_{M}+(1 / 4) k W_{M}^{2}\right)}{\left\{\lambda_{\min }(\mathbf{Q})+\lambda_{\min }\left(R^{-1}\right)\right\}} \leq\|\widetilde{\mathbf{z}}\|, \\
\sqrt{\frac{\varepsilon_{M}}{k}+\frac{1}{4} W_{M}^{2}}+\frac{1}{2} W_{M} \leq\|\widetilde{\mathbf{W}}\|_{F} .
\end{gathered}
$$

Thus, $\dot{L}_{3}$ is negative outside a compact set. According to a standard Lyapunov theory extension [28], this demonstrates uniform ultimate boundedness of $e(t), r(t)$, and $\widetilde{\mathbf{W}}(t)$.

\section{Simulation Results}

The drive cycle tests that are currently used for light-weighted EVs are new European driving cycle (NEDC), Federal Test Procedure (FTP-75), and JC08. The NEDC is used in Europe, and the low powered $\mathrm{EV}$ version of this cycle is used in India. The FTP 75 cycle is used in USA and the JC08 in Japan. In order to show the validity of the proposed controllers, the NEDC is used for testing the performance. The NEDC is a driving cycle consisting of four repeated ECE-15 driving cycles and an extra-urban driving cycle (EUDC) [1]. The maximum speed of NEDC is $120 \mathrm{~km} / \mathrm{h}$ but it is scaled to $50 \mathrm{~km} / \mathrm{h}$ when applied in this paper [11].

The simulation is implemented in MATLAB 7.8.0 (2009a) with $\mathrm{m}$-file programming. The controller design parameters for conventional backstepping are chosen as $\bar{k}_{1}=15$ and $\bar{k}_{2}=15$. The controller design parameters for CNN based backstepping are chosen as $k_{1}=0.8$ and $k_{2}=0.35$. For update of parameters in (48) and (49), $\eta_{1}, \eta_{2}$, and $\bar{\rho}$ are chosen as 1 , 0.0001 , and 0.15 , respectively. For approximating $f(x, z)$ the input to the CNN 1 is $z_{1}, z_{2}$, and for approximating $g(x, z)$ the input to the CNN 2 is $z_{1}, z_{2}$. The order of Chebyshev polynomial is chosen as 1 for both $z_{1}$ and $z_{2}$. The parameters of CNN are initialized to zero. Thus $\phi_{1}$ and $\phi_{2}$ have dimension $(5 \times 1)$, where $\phi_{1}=\phi_{2}=\left[\begin{array}{lllll}1 & z_{1} & 2 z_{1}^{2}-1 & z_{2} & 2 z_{2}^{2}-1\end{array}\right]^{T} \cdot \mathbf{W}_{1}$ and $\mathbf{W}_{2}$ have dimension of $(5 \times 1)$. For optimal controller the design parameters are chosen as $\Lambda=1.25, K=4$. For CNN based optimal adaptive controller parameter $k_{z}$ is chosen as 0.0001 . For update of parameters in (95), $\Gamma$ and $k$ are chosen as 0.01. For approximating $h(x)$ the input to the $\mathrm{CNN}$ is $z_{1}, z_{2}, e$, $y_{d}, \dot{y}_{d}$, and $\ddot{y}_{d}$. The order of Chebyshev polynomial is chosen as 1 for all inputs to the CNN. The parameters of CNN are initialized to zero. Thus $\varphi$ and $\mathbf{W}$ have dimension of $(13 \times 1)$. The initial conditions for $\left[z_{1}(0), z_{2}(0)\right]^{T}=[0.01,0.1]^{T}$.

Performance of designed controllers for mass variation as given in (106) is considered. Passengers mass is increased/decreased at different point of time in the driving cycle.The variation in armature winding resistance of the DC motor due to temperature changes, the variation in the aerodynamic drag coefficient, and the variation in the rolling resistance coefficient are considered as given in (107), (108), and (109), respectively. The variation in mass, armature winding resistance, aerodynamic drag coefficient, and rolling resistance coefficient are assumed to be known in conventional backstepping and optimal controller. The variation in mass, armature winding resistance, aerodynamic drag coefficient, and rolling resistance coefficient are considered unknown in CNN based backstepping controller and $\mathrm{CNN}$ based optimal adaptive controller.

Choosing

$$
\begin{gathered}
m= \begin{cases}950 & 0<t \leq 195 \\
1020 & 195<t \leq 585 \\
1080 & 585<t<780 \\
950 & 780 \leq t \leq 1180\end{cases} \\
R_{a}= \begin{cases}0.0867 & 300<t \leq 500 \\
0.0947 & 500<t \leq 780\end{cases} \\
C_{d}= \begin{cases}0.27 & 200<t \leq 300 \\
0.33 & 600<t \leq 780,\end{cases} \\
\mu_{r r}= \begin{cases}0.0165 & 200<t \leq 300 \\
0.0135 & 600<t \leq 780 .\end{cases}
\end{gathered}
$$

The drive cycle test performances and tracking errors for conventional backstepping controller and CNN based backstepping controller are shown in Figures 6 and 7, respectively. It is clear that the conventional backstepping controller has better tracking performance than the CNN based backstepping controller, in the range of speed below designed nominal speed $(v=25 \mathrm{~km} / \mathrm{hr})$. The CNN based backstepping controller performs much better in high speed range. The amp-hour consumption for $\mathrm{CNN}$ based backstepping controller is $4.4834 \mathrm{~km} / 10.75 \mathrm{AH}$.

The drive cycle test performances and tracking errors for optimal controller and $\mathrm{CNN}$ based optimal adaptive controller are shown in Figures 8 and 9, respectively. The amp-hour consumption for CNN based optimal adaptive controller is $4.4795 \mathrm{~km} / 10.70 \mathrm{AH}$. It can be seen that the optimal controller has better tracking performance in the speed 


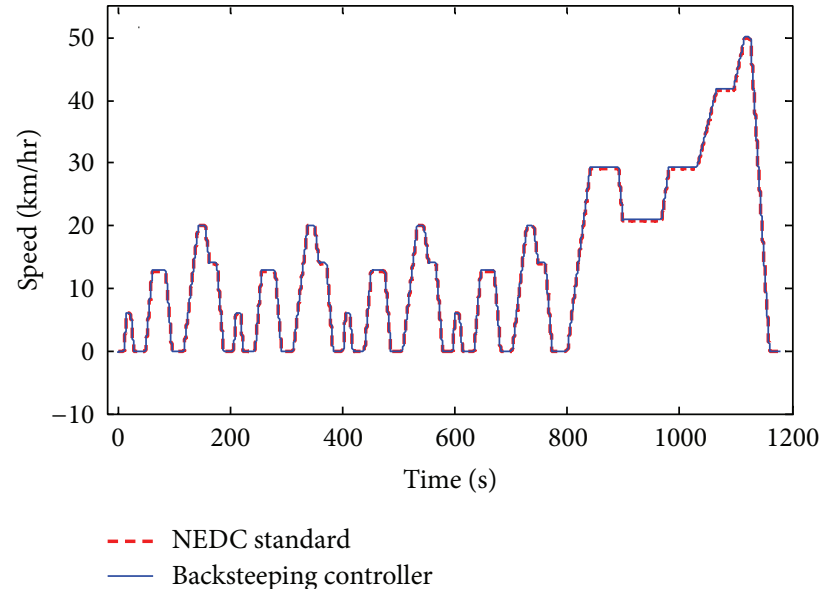

(a)

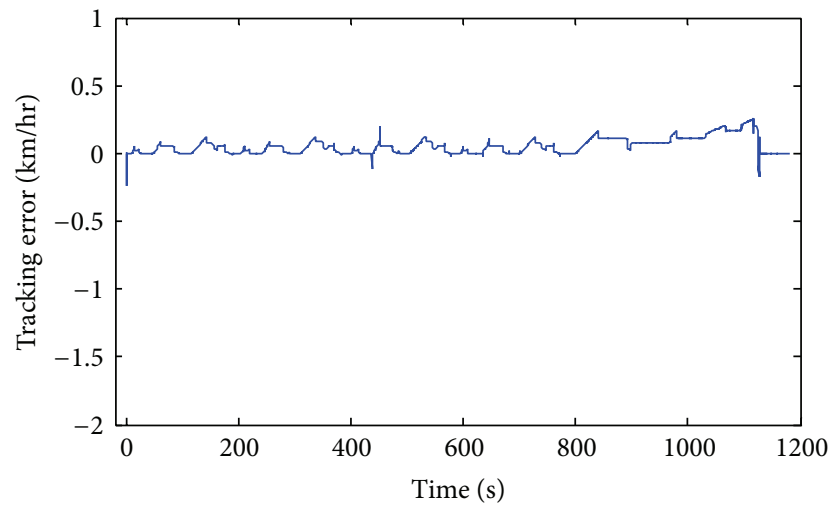

Figure 6: Performance and tracking error of conventional backstepping controller.

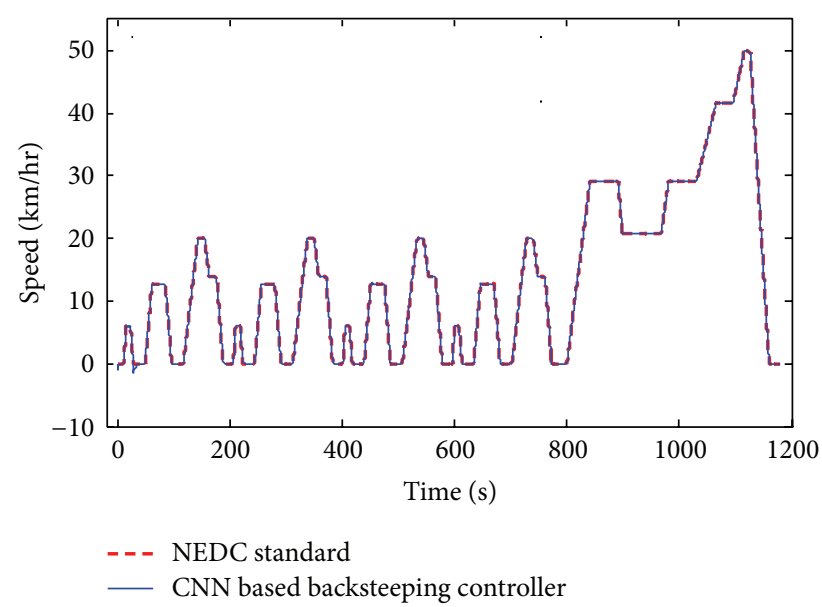

(a)

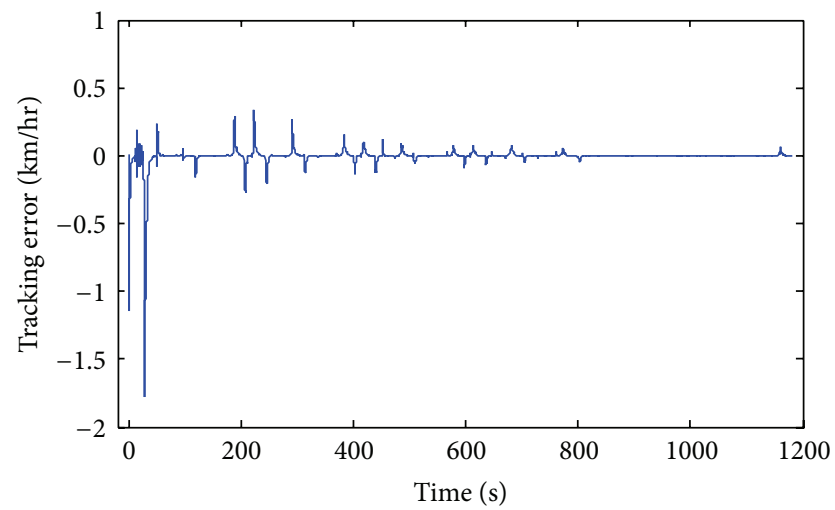

FIGURE 7: Performance and tracking error of CNN based backstepping controller.

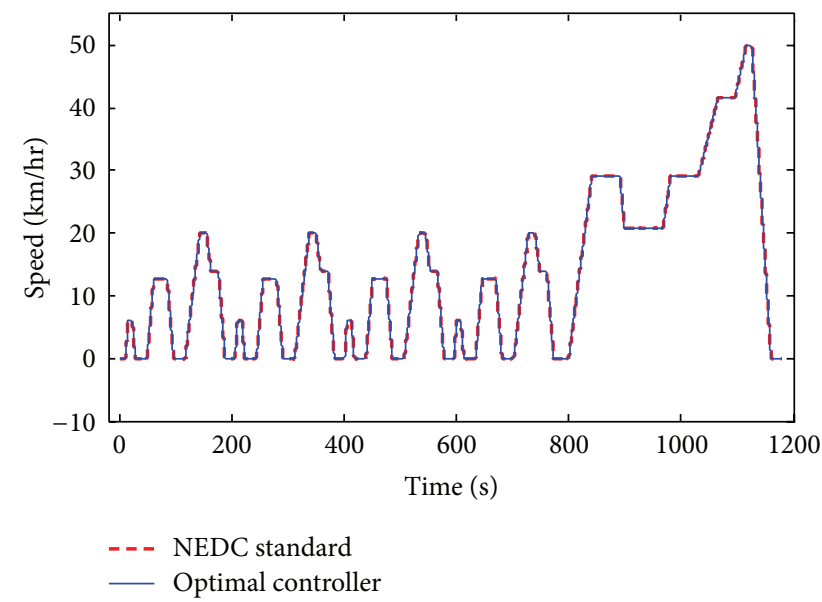

(a)

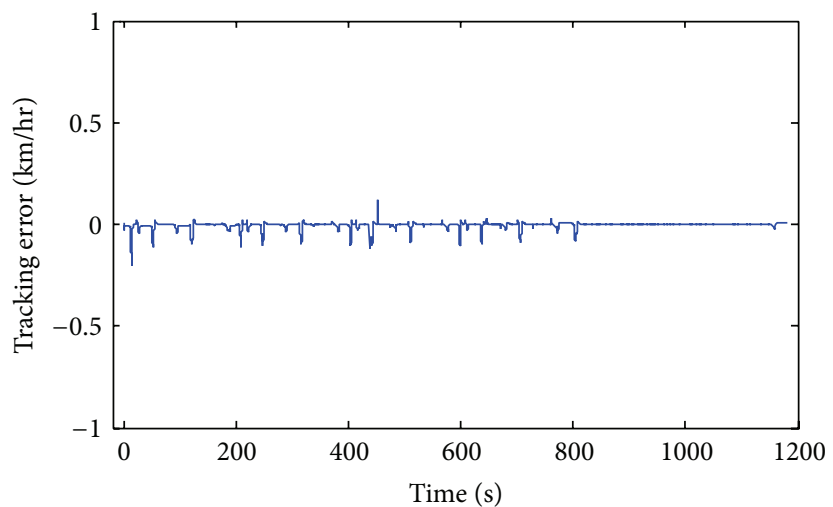

FIGURE 8: Performance and tracking error of optimal controller. 




(a)

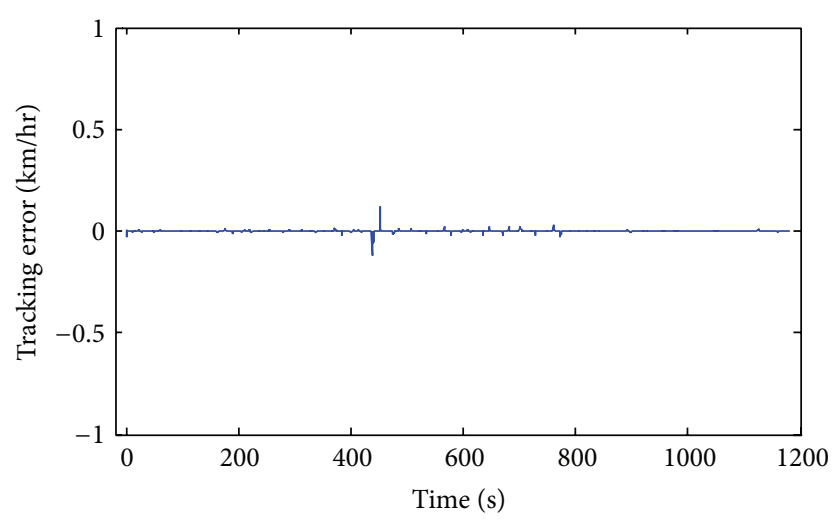

(b)

FIGURE 9: Performance and tracking error of CNN based optimal adaptive controller.

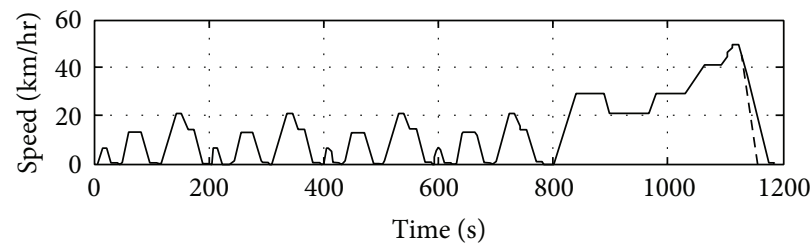

- Nonlinear optimal
- - NEDC standard

(a)

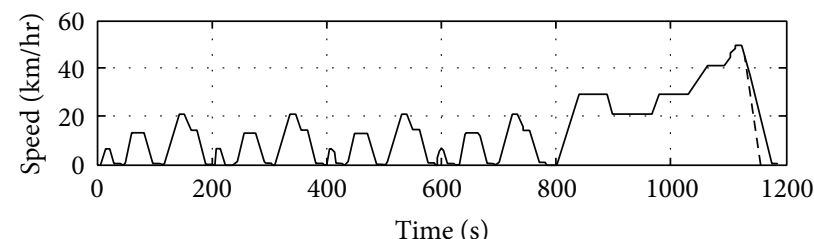

- Nonlinear robust

- - - NEDC standard

FIGURE 10: Results of NEDC test of [11].

TABLE 2: Comparative results of driving cycle test.

\begin{tabular}{lc}
\hline Controller & Amp-hour consumption \\
\hline Nonlinear optimal [11] & $4.48 \mathrm{~km} / 11.97 \mathrm{AH}$ \\
Nonlinear robust [11] & $4.4825 \mathrm{~km} / 10.78 \mathrm{AH}$ \\
CNN based backstepping & $4.4834 \mathrm{~km} / 10.75 \mathrm{AH}$ \\
CNN based optimal adaptive & $4.4795 \mathrm{~km} / 10.70 \mathrm{AH}$ \\
\hline
\end{tabular}

range below designed nominal speed than conventional backstepping controller and CNN based backstepping controller. However, for the high speed range the performance of CNN based backstepping controller is comparable to the optimal controller. CNN based optimal adaptive controller gives the best tracking performance on the entire speed range of the drive cycle test as compared to $\mathrm{CNN}$ based backstepping controller designed in current work and the results presented in [11] which are reproduced in Figure 10. The comparative result of the driving cycle test is presented in Table 2 and to provide detailed quantitative analysis of the designed controllers, root mean squared (RMS) tracking error of controllers is presented in Table 3 . The proposed $\mathrm{CNN}$ based optimal adaptive learning shows both robustness and adaptation to changing system dynamics and unknown nonlinearities.
TABLE 3: RMS tracking error of controllers designed.

\begin{tabular}{lc}
\hline Controller & RMS value of tracking errors \\
\hline Conventional backstepping & 0.0301 \\
CNN based backstepping & 0.0279 \\
Optimal & 0.0165 \\
CNN based optimal adaptive & 0.0040 \\
\hline
\end{tabular}

The traction force is required to propel the $\mathrm{EV}$ in forward direction. It is produced by the DC motor torque and transferred through transmission unit, which includes the gearing system and finally drive the vehicle. While the vehicle is in motion, there are forces that try to stop its movement. These forces usually include rolling resistance and aerodynamic drag force. The simulation result of traction force of the EV is shown in Figure 11.

In present work, all the parameters are specified on a light-weighted all-electric vehicle and are given in Table 1. The mass of the vehicle is considered as $800 \mathrm{~kg}$. This includes the body mass of the vehicle, accessories, weight of the DC motor, and batteries. For modeling light-weighted EVs, we have considered four batteries each of 12 volts and this restricts the control effort in the range of $0 \sim 48 \mathrm{~V}$ (see Table 1). The control effort is shown in Figure 12. 


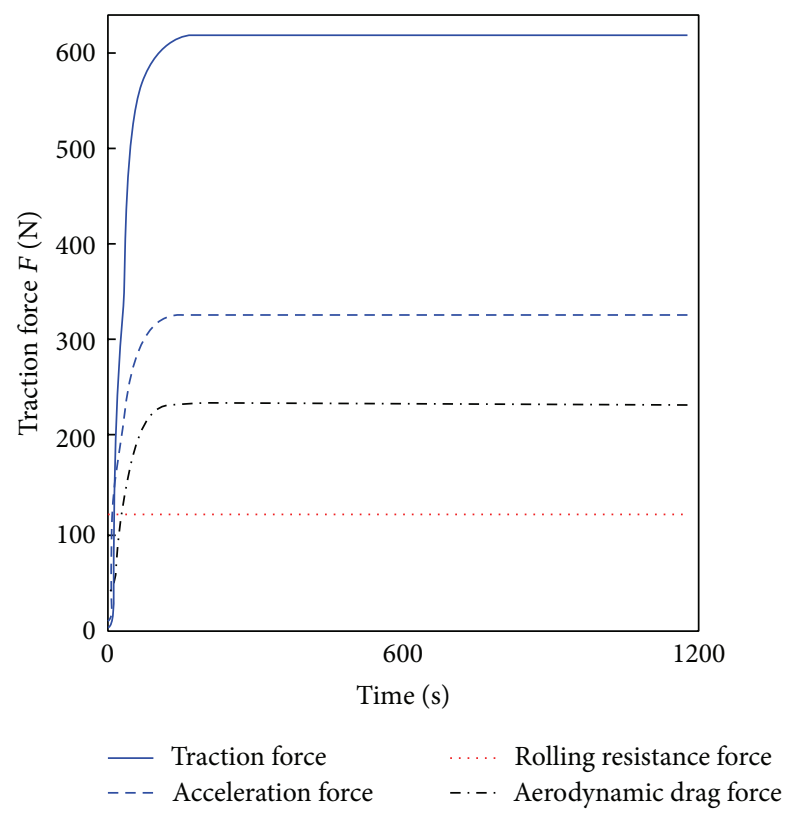

FIgURE 11: Traction force.

\section{Conclusion}

Two nonlinear controllers for a light-weighted all-electric vehicle, CNN based backstepping controller and CNN based optimal adaptive controller, are presented in this paper. The unknown nonlinearities in EV system arise due to varying mass of passengers, varying resistance in the armature winding of the DC motor and variation in aerodynamic drag coefficient and the rolling resistance coefficient are estimated by $\mathrm{CNN}$. The CNN weights are updated online according to the adaptive-learning algorithm, which is obtained from Lyapunov stability analysis, so that system-tracking stability and error convergence can be assured in the closed-loop system. The salient feature of the proposed design methodologies demonstrates that the control objective is obtained with unknown nonlinear dynamics of the EV system. The NEDC is used for testing the performance of the proposed controllers. It is shown that the tracking performance of the controllers designed in this paper is satisfactory in both the cases. The test results for CNN based backstepping controller and the CNN based optimal adaptive controller have better tracking performance than that reported by Huang et al. [11] and amp-hour consumption is also less than the nonlinear controllers described in [11]. From simulation results and the comparative and quantitative results presented in Tables 2 and 3, respectively, it is clear that the $\mathrm{CNN}$ based optimal adaptive controller gives better performance as compared to other controllers.

\section{Conflict of Interests}

The authors declare that there is no conflict of interests regarding the publication of this paper.

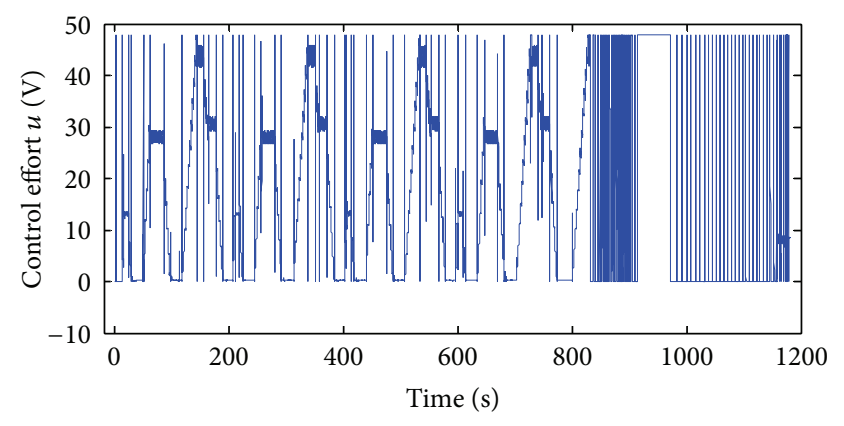

FIGURE 12: Control effort $u(t)$.

\section{Acknowledgment}

The authors would like to thank the editor and the anonymous reviewers for their valuable comments and constructive suggestions which have helped in improving the quality of the paper.

\section{References}

[1] J. Larminie and J. Lowry, Electric Vehicle Technology Explained, John Wiley \& Sons, West Sussex, UK, 2003.

[2] Y. Cheng, J. Van Mierlo, P. Van Den Bossche, and P. Lataire, "Energy sources control and management in hybrid electric vehicles," in Proceedings of the 12th International Power Electronics and Motion Control Conference (EPE-PEMC '06), pp. 524530, Portoroz, Slovenia, September 2006.

[3] D. F. Opila, X. Wang, R. McGee, R. B. Gillespie, J. A. Cook, and J. W. Grizzle, "An energy management controller to optimally trade off fuel economy and drivability for hybrid vehicles," IEEE Transactions on Control Systems Technology, vol. 20, no. 6, pp. 1490-1505, 2012.

[4] Y. L. Murphey, J. Park, Z. Chen, M. L. Kuang, M. A. Masrur, and A. M. Phillips, "Intelligent hybrid vehicle power control-part I: machine learning of optimal vehicle power," IEEE Transaction on Vehicular Technology, vol. 61, pp. 3519-3530, 2012.

[5] Y. L. Murphey, J. Park, L. Kiliaris et al., "Intelligent hybrid vehicle power control-part II: online intelligent energy management," IEEE Transaction on Vehicular Technology, vol. 62, pp. 69-79, 2013.

[6] H. Zhang, L. M. Tolbert, and B. Ozpineci, "Impact of SiC devices on hybrid electric and plug-in hybrid electric vehicles," IEEE Transactions on Industry Applications, vol. 47, no. 2, pp. 912-921, 2011.

[7] J. Dixon, I. Nakashima, E. F. Arcos, and M. Ortúzar, "Electric vehicle using a combination of ultracapacitors and ZEBRA battery," IEEE Transactions on Industrial Electronics, vol. 57, no. 3, pp. 943-949, 2010.

[8] M. Ye, Z. Bai, and B. Cao, "Robust control for regenerative braking of battery electric vehicle," IET Control Theory and Applications, vol. 2, no. 12, pp. 1105-1114, 2008.

[9] M. Pahlevaninezhad, P. Das, J. Drobnik, G. Moschopoulos, P. K. Jain, and A. Bakhshai, "A nonlinear optimal control approach based on the control-lyapunov function for an AC/DC converter used in electric vehicles," IEEE Transaction on Industrial Informatics, vol. 8, pp. 596-614, 2012. 
[10] R. Wang and J. Wang, "Passive actuator fault-tolerant control for a class of overactuated nonlinear systems and applications to electric vehicles," IEEE Transaction on Vehicular Technology, vol. 62, pp. 972-985, 2013.

[11] Q. Huang, Z. Huang, and H. Zhou, "Nonlinear optimal and robust speed control for a light-weighted all-electric vehicle," IET Control Theory and Applications, vol. 3, no. 4, pp. 437-444, 2009.

[12] S. Poorani, K. Udaya Kumar, and S. Renganarayanan, "Intelligent controller design for electric vehicle," in Proceedings of the 57th IEEE Semiannual Vehicular Technology Conference (VTC '03), vol. 4, pp. 2447-2450, Jeju, Korea, April 2003.

[13] P. Khatun, C. M. Bingham, N. Schofield, and P. H. Mellor, "Application of fuzzy control algorithms for electric vehicle antilock braking/traction control systems," IEEE Transactions on Vehicular Technology, vol. 52, no. 5, pp. 1356-1364, 2003.

[14] V. Schwarzer and R. Ghorbani, "Drive cycle generation for design optimization of electric vehicles," IEEE Transaction on Vehicular Technology, vol. 62, pp. 89-97, 2013.

[15] S. Kachroudi, M. Grossard, and N. Abroug, "Predictive driving guidance of full electric vehicles using particle swarm optimization," IEEE Transaction on Vehicular Technology, vol. 61, pp. 1309-3919, 2012.

[16] A. Namatame and N. Ueda, "Pattern classification with Chebyshev neural networks," International Journal of Neural Networks, vol. 3, pp. 23-31, 1992.

[17] T.-T. Lee and J.-T. Jeng, "The Chebyshev-polynomials-based unified model neural networks for function approximation," IEEE Transactions on Systems, Man, and Cybernetics B: Cybernetics, vol. 28, no. 6, pp. 925-935, 1998.

[18] J. C. Patra and A. C. Kot, "Nonlinear dynamic system identification using Chebyshev functional link artificial neural networks," IEEE Transactions on Systems, Man, and Cybernetics B: Cybernetics, vol. 32, no. 4, pp. 505-511, 2002.

[19] S. Purwar, I. N. Kar, and A. N. Jha, "On-line system identification of complex systems using Chebyshev neural networks," Applied Soft Computing Journal, vol. 7, no. 1, pp. 364-372, 2007.

[20] J. C. Patra, "Chebyshev neural network-based model for dualjunction solar cells," IEEE Transactions on Energy Conversion, vol. 26, no. 1, pp. 132-139, 2011.

[21] S. Purwar, I. N. Kar, and A. N. Jha, "Adaptive output feedback tracking control of robot manipulators using position measurements only," Expert Systems with Applications, vol. 34, no. 4, pp. 2789-2798, 2008.

[22] A.-M. Zou, K. D. Kumar, and Z.-G. Hou, "Quaternion-based adaptive output feedback attitude control of spacecraft using chebyshev neural networks," IEEE Transactions on Neural Networks, vol. 21, no. 9, pp. 1457-1471, 2010.

[23] A.-M. Zou, K. D. Kumar, Z.-G. Hou, and X. Liu, "Finite-time attitude tracking control for spacecraft using terminal sliding mode and chebyshev neural network," IEEE Transactions on Systems, Man, and Cybernetics B: Cybernetics, vol. 41, no. 4, pp. 950-963, 2011.

[24] D. Wang and J. Huang, "Neural network-based adaptive dynamic surface control for a class of uncertain nonlinear systems in strict-feedback form," IEEE Transactions on Neural Networks, vol. 16, no. 1, pp. 195-202, 2005.

[25] H. K. Khalil, Nonlinear Systems, Prentice Hall, Upper Saddle River, NJ, USA, 3rd edition, 2002.

[26] C. Kwan and F. L. Lewis, "Robust backstepping control of nonlinear systems using neural networks," IEEE Transactions on
Systems, Man, and Cybernetics A: Systems and Humans, vol. 30, no. 6, pp. 753-766, 2000.

[27] Y. H. Kim and F. L. Lewis, "Optimal design of CMAC neuralnetwork controller for robot manipulators," IEEE Transactions on Systems, Man and Cybernetics C: Applications and Reviews, vol. 30, no. 1, pp. 22-31, 2000.

[28] K. S. Narendra and A. M. Annaswamy, "A new adaptive law for robust adaptation without persistent excitation," IEEE Transactions on Automatic Control, vol. 32, no. 2, pp. 134-145, 1987.

[29] F. L. Lewis and V. L. Syrmos, Optimal Control, John Wiley \& Sons, New York, NY, USA, 2nd edition, 1995. 

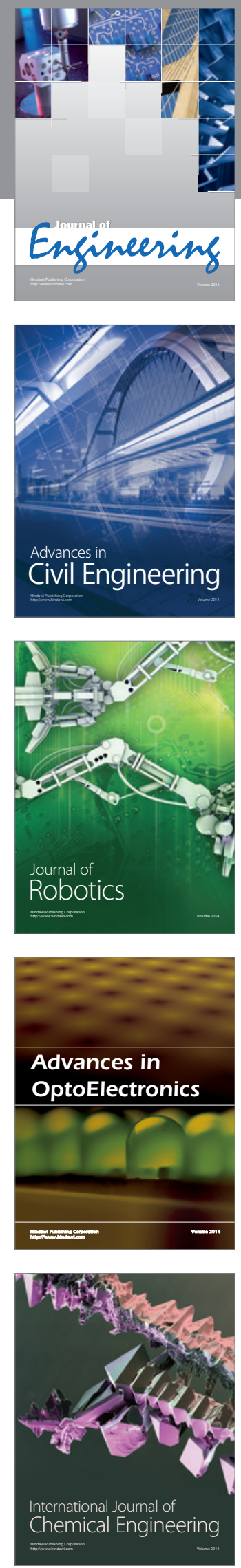

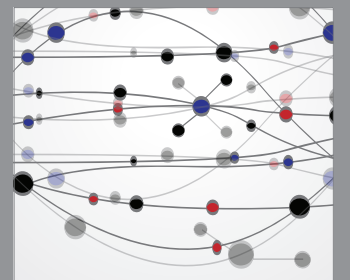

The Scientific World Journal
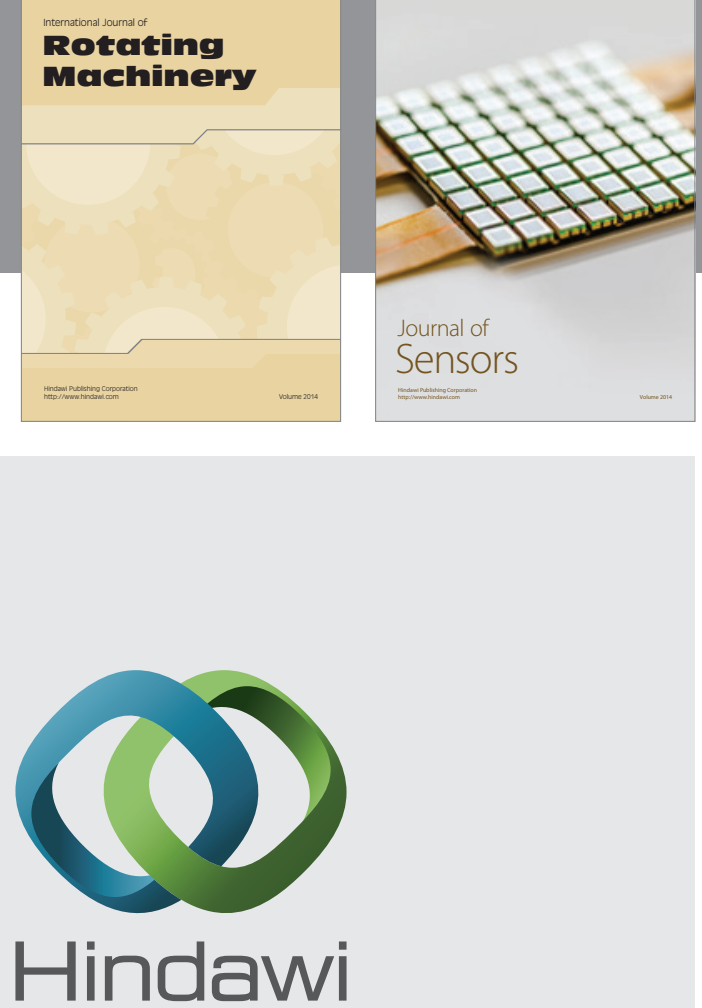

Submit your manuscripts at http://www.hindawi.com
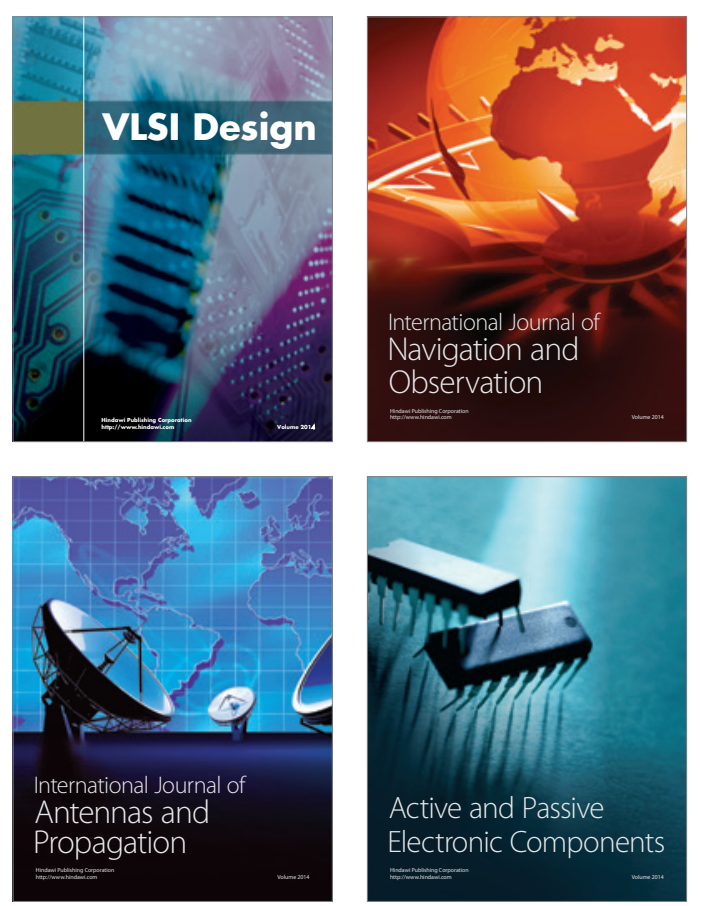
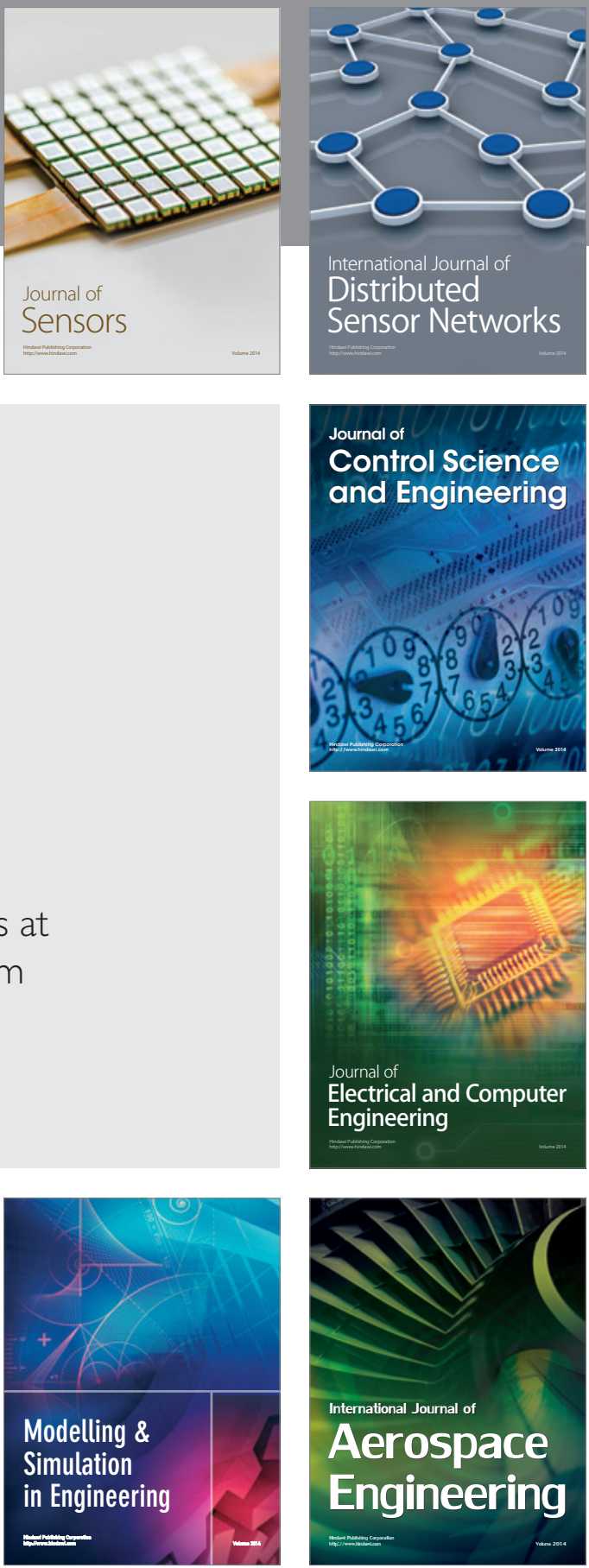

Journal of

Control Science

and Engineering
